Homology, Homotopy and Applications, vol.17(1), 2015, pp.203-234

\title{
TORSORS AND THE QUILLEN-BARR-BECK COHOMOLOGY FOR RESTRICTED LIE ALGEBRAS
}

\section{IOANNIS DOKAS}

\author{
(communicated by Claude Cibils)
}

\begin{abstract}
In this paper we study Duskin-Glenn torsor cohomology in the context of restricted Lie algebras. In particular, we give an interpretation of the torsor cohomology groups which appear in Cegarra-Aznar's eight-term exact sequence. Thus, we prove a classification theorem for the second Quillen-Barr-Beck cohomology in terms of 2-fold extensions of restricted Lie algebras.
\end{abstract}

This paper is dedicated to the memory of Jean-Louis Loday.

\section{Introduction}

First Hochschild in [16] and later Pareigis in [21] defined cohomology groups for the category RLie of restricted Lie algebras. Following the general scheme for a cohomology theory of universal algebras by Quillen [23] and Barr and Beck [2], in [9] it is considered a new approach for a cohomology theory of restricted Lie algebras. Thus, for $L \in \mathrm{RLie}$, any restricted Lie algebra, and $(A, f) \in a b(\mathrm{RLie} / L)$, any abelian group object in the comma category of restricted Lie algebras over $L$, cotriple cohomology groups $H_{\mathbf{G}}^{*}(L,(A, f))$ are studied. Both Pareigis and Quillen-Barr-Beck cohomology classify general abelian extensions of restricted Lie algebras, whereas Hochschild cohomology classifies strongly abelian extensions. The use of the Quillen-Barr-Beck method gives us the opportunity to define higher (co)homology groups in a natural way.

For the category of restricted Lie algebras, it is proved by Eckmann and Stammbach in $[\mathbf{1 2}]$ that there is a five-term exact sequence for Hochschild (co)homology. In the framework of a Barr-exact category, Cegarra and Aznar in [7] proved that there is an eight-term exact sequence in the first variable for Duskin-Glenn's torsor cohomology [10], [15].

In this paper we give, for the category of restricted Lie algebras, an interpretation of the torsor cohomology groups which appear in the Cegarra-Aznar sequence. In

I would like to express my appreciation to the Isaac Newton Institute for the excellent working conditions that I enjoyed there during the program "Grothendieck-Teichmüller groups, Deformations and Operads."

Received June 24, 2013, revised March 17, 2014, October 6, 2014, November 4, 2014; published on April 16, 2015.

2010 Mathematics Subject Classification: 18C15, 18G30, 17B55, 17B50.

Key words and phrases: restricted Lie algebra, Quillen-Barr-Beck cohomology, torsor, crossed module.

Article available at http://dx.doi.org/10.4310/HHA.2015.v17.n1.a10

Copyright (C) 2015, International Press. Permission to copy for private use granted. 
particular, in Lemma 3.1 of Section 3 we compute the torsor 0-cohomology group of an epimorphism. In Section 4, we show an equivalence between the category of crossed modules of restricted Lie algebras and the category of internal groupoids in RLie, a result which we apply in Section 5 to prove that there is an isomorphism between the torsor 2-cohomology groups $\operatorname{Tors}^{2}(L,(A, f))$ and the abelian groups $\operatorname{Ext}_{p}^{2}(L,(A, f))$ of 2 -fold extensions of restricted Lie algebras. Thus we are able to obtain a classification theorem for the second Quillen-Barr-Beck cohomology in terms of 2-fold extensions. As a consequence of these interpretations, from the CegarraAznar sequence we obtain that for an exact sequence of restricted Lie algebras $0 \rightarrow$ $N \rightarrow E \stackrel{\phi}{\rightarrow} L \rightarrow 0$, there are naturally equivalent exact sequences

$$
\begin{gathered}
0 \rightarrow \operatorname{Der}_{p}(L,(A, f)) \rightarrow \operatorname{Der}_{p}(E,(A, f)) \rightarrow \operatorname{Hom}_{w(L)}\left(\left(N_{a b},(-)^{[p]}\right),(A, f)\right) \rightarrow \\
\operatorname{Ext}_{p}^{1}(L,(A, f)) \rightarrow \rightarrow \operatorname{Ext}_{p}^{1}(E,(A, f)) \rightarrow \operatorname{Ext}_{p}^{2}(\phi,(A, f)) \rightarrow \\
\operatorname{Ext}_{p}^{2}(L,(A, f)) \rightarrow \operatorname{Ext}_{p}^{2}(E,(A, f)) \\
0 \rightarrow H_{\mathbf{G}}^{0}(L,(A, f)) \rightarrow H_{\mathbf{G}}^{0}(E,(A, f)) \rightarrow \operatorname{Hom}_{w(L)}\left(\left(N_{a b},(-)^{[p]}\right),(A, f)\right) \rightarrow \\
H_{\mathbf{G}}^{1}(L,(A, f)) \rightarrow \rightarrow H_{\mathbf{G}}^{1}(E,(A, f)) \rightarrow \operatorname{Ext}_{p}^{2}(\phi,(A, f)) \rightarrow \\
H_{\mathbf{G}}^{2}(L,(A, f)) \rightarrow H_{\mathbf{G}}^{2}(E,(A, f))
\end{gathered}
$$

which replace Eckmann-Stammbach's five-term exact sequence in the context of Quillen-Barr-Beck cohomology. Since any extension of algebraic groups induces a short exact sequence of restricted Lie algebras, we get an application in the theory of algebraic groups in prime characteristic.

\section{Preliminaries}

\subsection{Restricted Lie algebras}

In modular Lie theory, in order to extend theorems which are valid in characteristic zero we are led to consider restricted Lie algebras introduced by N. Jacobson in [18]. Let $k$ denote a field of characteristic $p \neq 0$ and Lie the category of Lie algebras over $k$.

Definition 2.1. A restricted Lie algebra $L=\left(L,(-)^{[p]}\right)$ over $k$ is a Lie algebra $L \in$ Lie together with a map $(-)^{[p]}: L \rightarrow L$ called the $p$-map such that the following relations hold:

$$
\begin{aligned}
(\alpha x)^{[p]} & =\alpha^{p} x^{[p]} \\
{\left[x, y^{[p]}\right] } & =[\cdots[[x, \underbrace{y], y], \cdots, y}_{p}] \\
(x+y)^{[p]} & =x^{[p]}+y^{[p]}+\sum_{i=1}^{p-1} s_{i}(x, y)
\end{aligned}
$$

where $i s_{i}(x, y)$ is the coefficient of $\lambda^{i-1}$ in $a d_{\lambda x+y}^{p-1}(x)$ and $a d_{x}: L \rightarrow L$ denotes the adjoint representation given by $a d_{x}(y):=[y, x], x, y \in L, \alpha \in k$. A Lie algebra homomorphism $f: L \rightarrow L^{\prime}$ is called restricted if $f\left(x^{[p]}\right)=f(x)^{[p]}$. We denote by RLie the category of restricted Lie algebras over $k$. 
Remark 2.2. Let $L \in \mathrm{RLie}$ be a restricted Lie algebra and $x, y \in L$. If $L_{x, y}$ is the Lie algebra generated by the elements $x, y$, then for $0 \leqslant i \leqslant p-1, s_{i}(x, y) \in L_{x, y}^{p}$, where $L_{x, y}^{1}:=L_{x, y}$ and

$$
L_{x, y}^{n}:=\left[L_{x, y}^{n-1}, L_{x, y}\right]
$$

Remark 2.3. There is a notion of free restricted Lie algebra over a set. Therefore the category RLie of restricted Lie algebras is a monadic category over Sets. It follows that RLie is a Barr exact category.

Let $L$ and $L^{\prime}$ be restricted Lie algebras. The direct product of $L$ and $L^{\prime}$ is, as a Lie algebra, their direct product in the category of Lie algebras

$$
L \times L^{\prime}=\left\{\left(x, x^{\prime}\right): x \in L, x^{\prime} \in L^{\prime}\right\}
$$

equipped with the $p$-map given by $\left(x, x^{\prime}\right)^{[p]}:=\left(x^{[p]}, x^{\prime[p]}\right)$ for all $\left(x, x^{\prime}\right) \in L \times L^{\prime}$. If $f: L \rightarrow R$ and $f^{\prime}: L^{\prime} \rightarrow R$ are restricted Lie homomorphisms, then the pullback of $L$ and $L^{\prime}$ over $R$ is given by the following commutative diagram in RLie:

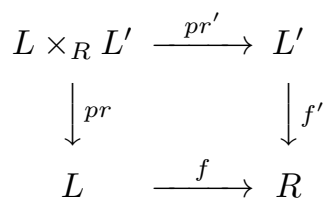

where

$$
L \times{ }_{R} L^{\prime}:=\left\{\left(x, x^{\prime}\right) \in L \times L^{\prime}: f(x)=f^{\prime}\left(x^{\prime}\right)\right\} \leqslant L \times L^{\prime}
$$

Example 2.4. Let $\mathfrak{A}$ be any associative algebra over a field $k$ with characteristic $p \neq 0$. We denote by $\mathfrak{A}_{\text {Lie }}$ the induced Lie algebra with the bracket given by $[x, y]:=x y-y x$, for all $x, y \in \mathfrak{A}$. Then $\left(\mathfrak{A},(-)^{p}\right)$ is a restricted Lie algebra where $(-)^{p}$ is the Frobenious map given by $x \mapsto x^{p}$. Thus there is a functor $(-)_{\text {RLie }}:$ As $\rightarrow$ RLie from the category of associative algebras to the category of restricted Lie algebras.

Example 2.5. Let $\mathfrak{A}$ be an associative algebra over $k$. Then $\mathfrak{g l}_{n}(\mathfrak{A})$, the Lie algebra of $n \times n$ matrices with coefficients in $\mathfrak{A}$, is a restricted Lie algebra.

Example 2.6. If $V$ is a $k$-vector space, then a map $f: V \rightarrow V$ such that $f(x+y)=$ $f(x)+f(y)$ and $f(\alpha x)=\alpha^{p} f(x)$ for all $x, y \in V$ and $\alpha \in k$ is called $p$-semi-linear. Any pair $(V, f)$ where $V$ is $k$-vector space and $f: V \rightarrow V$ a $p$-semi-linear map is an abelian restricted Lie algebra.

Example 2.7. If $\mathfrak{B}$ is a $k$-algebra, that is not necessarily associative, then the set of $k$-derivations $\operatorname{Der}(\mathfrak{B})$ is endowed with the structure of a restricted Lie algebra. In particular, if $D \in \operatorname{Der}(\mathfrak{B})$ by the Leibniz formula, then we have

$$
D^{p}(x y)=\sum_{i=0}^{i=p}\left(\begin{array}{c}
p \\
i
\end{array}\right) D^{i}(x) D^{p-i}(y)
$$

for all $x, y \in \mathfrak{B}$. Since the char $k=p$, we have $\left(\begin{array}{l}p \\ i\end{array}\right)=0$ for $1 \leqslant i \leqslant p-1$; therefore $D^{p} \in \operatorname{Der}(\mathfrak{B})$.

If $(L,[p]) \in \mathrm{RLie}$ is any restricted Lie algebra, then a derivation $D \in \operatorname{Der}(L)$ is called a restricted derivation if $D\left(x^{[p]}\right)=a d_{x}^{p-1}(D(x))$ for all $x \in L$. 
Example 2.8. Let $\mathrm{G}$ be an algebraic group over $k$. The associated Lie algebra Lie(G) of $\mathrm{G}$ is endowed with the structure of a restricted Lie algebra (see [4], [30]).

Suppose that $L$ and $N$ are two restricted Lie algebras, and that $\eta: L \rightarrow \operatorname{Der}(N)$ is any given restricted homomorphism such that $\eta(x)$ is a restricted derivation for every $x \in L$. Then, recall that the Lie product on the semi-direct product of $L$ and $N$ is given by

$$
\left[(l, n),\left(l^{\prime}, n^{\prime}\right)\right]=\left(\left[l, l^{\prime}\right], \eta(l) n^{\prime}-\eta\left(l^{\prime}\right) n+\left[n, n^{\prime}\right]\right)
$$

It follows from Jacobson's Theorem 11 in $[\mathbf{1 8}]$ that the semi-direct product of $L$ and $N$ is endowed with a $p$-map extending the $p$-maps on $L$ and $N$ (cf. Theorem 2.5 in $[\mathbf{2 8}]$ ). We call this restricted Lie algebra the semi-direct product of $L$ and $N$ in the category of restricted Lie algebras, and we denote it by $L \rtimes N$.

Let us recall below some definitions and results which we use in the next section. We refer the reader to $[\mathbf{9}]$ for details.

Let $L \in$ RLie be a restricted Lie algebra and $U(L)$ its enveloping algebra. We denote by $u(L):=U(L) /<x^{p}-x^{[p]}, x \in L>$ the restricted enveloping algebra of $L$. An $L$-module $A$ is called restricted if $\left.\left.\left.x^{[p]} a=(\underbrace{x(x(\cdots(x}_{p} a) \cdots\right)\right)\right)$. The category of restricted Lie $L$-modules is equivalent to the category of $u(L)$-modules.

\subsection{Beck derivations for RLie}

Let $L^{\prime} \in \mathrm{RLie} / L$ be a restricted Lie algebra over $L$, and $(A, f)$ a pair where $A$ is a restricted $L$-module, and $f: A \rightarrow A^{L}$ a $p$-semi-linear map, where $A^{L}=\{a \in A$ : $x a=0$ for all $x \in L\}$. Then we define

$$
\operatorname{Der}_{p}\left(L^{\prime},(A, f)\right):=\{d \in \operatorname{Der}\left(L^{\prime}, A\right): d\left(x^{[p]}\right)=\underbrace{x \cdots x}_{p-1} d x+f(d(x)), x \in L^{\prime}\}
$$

Since $f$ is $p$-semi-linear, $\operatorname{Der}_{p}\left(L^{\prime},(A, f)\right)$ is an abelian group under addition called the group of Beck derivations. We denote by $L \times_{f} A$ the semi-direct product in RLie of $L$ and $A$; that is, $L \times_{f} A$ is the semi-direct product in Lie together with the $p$-map

$$
(l, a)^{[p]}=(l^{[p]}, \underbrace{l \cdots l}_{p-1} a+f(a))
$$

where $x \in L$ and $a \in A$. It is proved in [9, Lemma 1.4] that there is an isomorphism

$$
\operatorname{Hom}_{\mathrm{RLie} / L}\left(L^{\prime}, L \times_{f} A\right) \simeq \operatorname{Der}_{p}\left(L^{\prime},(A, f)\right)
$$

given by

$$
\omega \mapsto p r_{A} \omega
$$

where $p r_{A}$ denotes the canonical projection. In his doctoral dissertation, Beck incorporates the various notions of module over enveloping algebras to the general notion of a Beck module. 


\subsection{Beck modules for RLie}

For $L \in$ RLie a restricted Lie algebra the next theorem gives a characterization of the category of abelian group objects of the slice category RLie/L, i.e., the category of Beck $L$-modules in RLie.

Theorem 2.9. The category of abelian group objects ab $(\mathrm{RLie} / L)$ is equivalent to the category $\mathcal{A}$ whose objects are pairs $(A, f)$ where $A$ is a restricted $L$-module and $f$ : $A \rightarrow A^{L}$ is a p-semi-linear map from $A$ into its submodule of invariants $A^{L}$ and whose morphisms $\left(A_{1}, f_{1}\right) \rightarrow\left(A_{2}, f_{2}\right)$ are $L$ homomorphisms $\alpha: A_{1} \rightarrow A_{2}$ such that $f_{2} \alpha=\alpha f_{1}$.

Proof. By isomorphism (4) it follows that $L \times_{f} A$ is an abelian group object in the slice category RLie/L. Conversely, by Lemma 1.6 in $[\mathbf{9}]$ it follows that any abelian group object $L^{\prime} \in a b(\mathrm{RLie} / L)$ is isomorphic to a restricted Lie algebra of the form $L \times_{f} A$ for a well determined pair $(A, f)$, where $A$ is a restricted $L$-module and $f: A \rightarrow A^{L}$ a $p$-semi-linear map.

Let $R_{f}$ be the polynomial ring consisting of the set of polynomials $\sum_{i=0}^{i=m} a_{i} f^{i}$ where $a_{i} \in k, f$ an indeterminate, and $f a=a^{p} f$. We denote by $w(L)$ the ring which as $k$-vector space is $w(L):=R_{f} \otimes_{k} u(L)$ and such that $R_{f} \rightarrow w(L)$ and $u(L) \rightarrow w(L)$ are algebra homomorphisms and

$$
(P \otimes 1)(1 \otimes l):=P \otimes l \text { and }(l \otimes 1)(P \otimes 1):=0
$$

for all $P \in R_{f}$ and $l \in L$. Since it is easy to see that a pair $(A, f)$ as in Theorem 2.9 is the same as a $w(L)$-module, we have another characterization of the category of abelian group objects $a b(\mathrm{RLie} / L)$.

Theorem 2.10. The category of abelian group objects ab(RLie/L) is equivalent to the category of $w(L)$-modules.

Proof. Theorem 1.8 in [9].

Hereafter, we identify a $w(L)$-module with a pair $(A, f)$. Cartan and Eilenberg in their book [6] describe a general context for the definition of (co)homology groups for various algebraic structures. In each case, an appropriate notion of enveloping algebra is used and the definition is given in terms of Ext and Tor functors. In this context, Hochschild in [16] and B. Pareigis in [21] defined (co)homology groups for the category of restricted Lie algebras. Moreover, cohomology as a derived functor of derivations for several algebraic categories is studied by Barr and Rinehart in [3]. Besides, D. Quillen in [24], [23] develops an axiomatic homotopy theory and cohomology groups in the context of model categories are defined. Through this development (co)homology groups for universal algebras are introduced (see section 2 in [23]). In [9], Quillen-Barr-Beck cohomology for the category of restricted Lie algebras is defined. Moreover, in the papers [2], [1] the relation between cotriple cohomology groups and the cohomology theories described in the Cartan-Eilenberg context is given. It is proved that for the categories of groups, associative algebras, and Lie algebras the two theories coincide (considering a shift in dimension). In contrast to the cases of other algebraic categories, Quillen-Barr-Beck cohomology for restricted Lie algebras does not coincide with Hochschild cohomology. Quillen-Barr-Beck cohomology and Pareigis cohomology classify general abelian extensions of restricted Lie 
algebras, whereas Hochschild cohomology classifies strongly abelian extensions, i.e., extensions such that the $p$-map on the kernel is zero. The Quillen-Barr-Beck method provides the tools to define higher (co)homology groups in a natural way.

\subsection{Quillen-Barr-Beck cohomology for RLie}

Let $L \in$ RLie be a restricted Lie algebra and $(A, f)$ a $w(L)$-module. Let $F:$ Sets $\rightarrow$ RLie be the free functor, left adjoint to the forgetful functor $U:$ RLie $\rightarrow$ Sets. The adjunction $(F, U)$ gives rise to a cotriple $\mathbf{G}$ in RLie/L. In [9] cotriple cohomology groups are defined:

$$
H_{\mathbf{G}}^{n}(L,(A, f)):=H^{n}\left(\operatorname{Der}_{p}\left(\mathbf{G}_{*}(L),(A, f)\right)\right)
$$

where $\mathbf{G}_{*}(L)$ denotes the cotriple resolution of $L$ associated to the cotriple $\mathbf{G}$. Below we recall the definition of an $n$-torsor; for details and terminology we refer the reader to $[\mathbf{1 0}],[\mathbf{1 1}]$, and $[\mathbf{1 5}]$.

\subsection{Torsors, interpretation of cotriple cohomology}

Duskin in [10] gave an interpretation of the cotriple cohomology in terms of $n$ dimensional torsors, generalizing to any dimension Beck's interpretation of dimension 1. Let $\mathrm{E}$ be a monadic category over Sets and $\mathbf{G}=F U$ the associated cotriple. The $n$-truncating functor

$$
t r^{n}: \operatorname{Simpl}(\mathrm{E}) \rightarrow t r^{n} \operatorname{Simpl}(\mathrm{E})
$$

from the category of simplicial objects of $\mathrm{E}$ to the category of $n$-truncated simplicial objects admits a right adjoint

$$
\operatorname{cosk}^{n}: t r^{n} \operatorname{Simpl}(\mathrm{E}) \rightarrow \operatorname{Simpl}(\mathrm{E})
$$

called Verdier's coskeleton functor. The coskeleton functor is constructed by iterating simplicial kernels. We denote by $\operatorname{Cosk}^{n}$ the composition functor

$$
\operatorname{Cosk}^{n}:=\operatorname{cosk}^{n} \circ \operatorname{tr}^{n}: \operatorname{Simpl}(\mathrm{E}) \rightarrow \operatorname{Simpl}(\mathrm{E})
$$

Let $A \in a b(\mathrm{E})$ be an abelian group object; then the simplicial object $K(A, n)$ is defined as the $(n+1)$-coskeleton of the following $(n+1)$-truncated simplicial object:

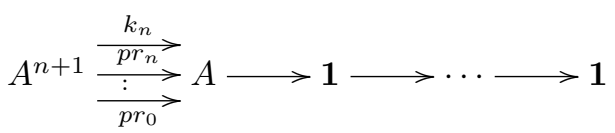

where 1 denotes the terminal object and $k_{n}=(-1)^{n} \sum_{i=0}^{i=n}(-1)^{i} p r_{i}$ for all $n \geqslant 1$. Let $X .=\left(\left(X_{j}\right)_{j \geqslant 0}, d_{i}, s_{i}\right)$ be a simplicial object and $\Lambda^{i}(m-1)(X$. $)$ the $i$-horn object of the $(m-1)$-truncated simplicial complex. Then there is a canonical map

$$
\left(d_{0}, \cdots, \hat{d}_{i}, \cdots, d_{m}\right): X_{m} \rightarrow \Lambda^{i}(m-1)(X .)
$$

Definition 2.11 (Duskin). Let $X \in \mathrm{E}$; then a $K(A, n)$-torsor in $\mathrm{E}$ over $X$ relative to $U$ is defined as an augmented over $X$ simplicial object $\left.X .=\left(\left(X_{j}\right)_{j \geqslant 0}\right), d_{i}, s_{i}\right)$ together with a simplicial morphism $\chi_{.}: X . \rightarrow K(A, n)$ such that:

1. $X . \rightarrow X$ is a $U$-split augmented simplicial object. 
2. $\chi .: X . \rightarrow K(A, n)$ is a simplicial morphism such that the following squares are pullbacks, for each $m \geqslant n$ and all $0 \leqslant i \leqslant m$ :

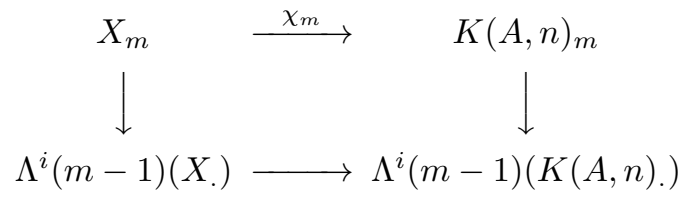

where the no-named maps are the canonical ones.

3. The canonical map $X$. $\rightarrow \operatorname{Cosk}^{n-1}(X$.) is an isomorphism.

If $(X, \chi$.$) and \left(X^{\prime}, \chi^{\prime}\right)$ are two $K(A, n)$-torsors over $X$, then a morphism of $K(A, n)$ torsors is an $X$-map $f .: X . \rightarrow X^{\prime}$ of augmented simplicial objects such that $\chi .=\chi^{\prime} f$. A $K(A, n)$-torsor over $X$ is referred to as an $n$-torsor over $X$ under $A$. The set of the connected components of $n$-torsors is denoted by $\operatorname{Tors}^{n}(X, A)$. If $X \in \mathrm{E}$, then it follows from (4.2) in [10] that an equivalent definition of $n$-torsor in the category $\mathrm{E} / \mathrm{X}$ is the following.

Definition 2.12 (Duskin). Let $X \in \mathrm{E}$ and $A \in a b(\mathrm{E} / \mathrm{X})$; then a $K(A, n)$-torsor over $X$ relative to $U$ is an augmented over $X$ simplicial object $X .=\left(\left(X_{j}\right)_{j \geqslant 0}, d_{i}, s_{i}\right)$ together with a morphism $\chi: X_{n} \rightarrow A$ over $X$ such that:

1. $X . \rightarrow X$ is a $U$-split augmented simplicial object.

2. $\chi$ is a normalized cocycle, that is, $\sum_{i=0}^{i=n+1}(-1)^{i} \chi d_{i}=0$ and $\chi s_{i}=0,0 \leqslant i \leqslant$ $n-1$.

3. If $s_{n}: X_{n-1} \rightarrow X_{n}$ is the $n$-th component map of the $U$-splitting, then on the underlying object level the commutative square

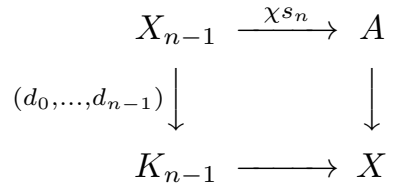

is a pullback, where $K_{n-1}$ is the $(n-2)$-simplicial kernel.

4. The canonical map $X$. $\rightarrow \operatorname{Cosk}^{n-1}(X$.) is an isomorphism.

Theorem 2.13. For any object $X \in \mathrm{E}$, and any abelian group object $A \in a b(\mathrm{E} / \mathrm{X})$, there is a natural bijection

$$
H_{\mathbf{G}}^{n}(X, A) \simeq \operatorname{Tors}^{n}(X, A),
$$

where $H_{\mathbf{G}}^{n}(X, A)$ is the $n$-th cotriple cohomology group of $X$ with coefficients in $A$.

Proof. Theorem (8.9) in $[\mathbf{1 0}]$.

Glenn in [15] defined the notion of $n$-dimensional hypergroupoid and gave a slightly different definition of $n$-torsor. If $\mathrm{E}$ is a monadic category over Sets whose objects have an underlying group structure, then by Corollary 7.2.4 in [15] the two notions of $n$-torsors coincide. Let $\phi: B \rightarrow R$ be a regular epimorphism in $\mathrm{E}$ and $B_{*}^{p}$ the simplicial object (with augmentation $\phi$ ), obtained by iterating the simplicial kernel 
construction. Then, A.M. Cegarra and E.R. Aznar in [7] define (7, Definition 1.1) the abelian groups

$$
\operatorname{Tors}^{0}(\phi, A):=\operatorname{Hom}_{\operatorname{simpl}(\mathrm{E})}\left(B_{*}^{p}, K(A, 1)\right)
$$

and $\operatorname{Tors}^{1}(\phi, A)$ of connected components of 2-torsors over $R$ with fixed augmentation $\phi(\mathbf{7}$, Definition 2.1). Moreover, it is proved in [7, Theorem 7.3] that there is an eight-term exact sequence

$$
\begin{aligned}
\operatorname{Hom}_{\mathrm{E}}(R, A) \stackrel{\phi^{*}}{\rightarrow} \operatorname{Hom}_{\mathrm{E}}(B, A) & \rightarrow \operatorname{Tors}^{0}(\phi, A) \rightarrow \operatorname{Tors}^{1}(R, A) \rightarrow \operatorname{Tors}^{1}(B, A) \\
& \rightarrow \operatorname{Tors}^{1}(\phi, A) \rightarrow \operatorname{Tors}^{2}(R, A) \rightarrow \operatorname{Tors}^{2}(B, A) .
\end{aligned}
$$

\section{A 5-term exact sequence for Quillen-Barr-Beck cohomology}

The Hochschild-Serre spectral sequence for (co)homology of Lie algebras gives rise to exact sequences of terms of low degree. In particular, if

$$
0 \rightarrow N \rightarrow E \rightarrow L \rightarrow 0
$$

is an exact sequence of Lie algebras and $A$ is a left (resp. right) $L$-module, then we have the following exact sequences:

$$
0 \rightarrow \operatorname{Der}(L, A) \rightarrow \operatorname{Der}(E, A) \rightarrow \operatorname{Hom}_{U(E)}\left(\mathrm{N}_{a b}, A\right) \rightarrow H^{2}(L, A) \rightarrow H^{2}(E, A)
$$

and

$$
H_{2}(E, A) \rightarrow H_{2}(L, A) \rightarrow \mathrm{N}_{a b} \otimes_{U(L)} A \rightarrow H_{1}(E, A) \rightarrow H_{1}(L, A) \rightarrow 0
$$

where $\mathrm{N}_{a b}:=N /[N, N]$.

For the case of Hochschild (co)homology of restricted Lie algebras, there are analogue sequences. Precisely, if

$$
0 \rightarrow N \rightarrow E \rightarrow L \rightarrow 0
$$

is an exact sequence of restricted Lie algebras and $M$ is a right restricted $\mathbf{b}$-module, then B. Eckmann and U. Stammbach proved in [12] the existence of the following 5-term exact sequence:

$$
H_{2}^{H o c h}(E, A) \rightarrow H_{2}^{H o c h}(L, A) \rightarrow \mathcal{N}_{a b} \otimes_{u(L)} A \rightarrow H_{1}^{H o c h}(E, A) \rightarrow H_{1}^{H o c h}(L, A) \rightarrow 0
$$

where $\mathcal{N}_{a b}:=N /[N, N]^{\prime}$ and $[N, N]^{\prime}$ denotes the ideal generated by the elements $[x, y], z^{[p]}$, where $x, y, z \in N$.

If $N$ is a restricted Lie algebra we denote by $N_{a b}:=N /[N, N]_{p}$ the quotient restricted Lie algebra, where $[N, N]_{p}$ is the $p$-ideal generated by the elements $[x, y]$, where $x, y \in N$. If $\phi: E \rightarrow L$ is a restricted Lie epimorphism with kernel $N$, then the $p$-map on $N$ induces an $R_{f}$-action on $N_{a b}$ given by

$$
f \cdot\left(n+[N, N]_{p}\right):=n^{[p]}+[N, N]_{p}
$$

Besides, $N_{a b}$ is an $L$-module via the action

$$
x \cdot\left(n+[N, N]_{p}\right):=[s(x), n]+[N, N]_{p}
$$


where $s: L \rightarrow E$ denotes a (any) set-theoretic section of $\phi$ and $x \in L, n \in N$. Moreover,

$$
\begin{aligned}
x \cdot\left(n^{[p]}+[N, N]_{p}\right) & =\left[s(x), n^{[p]}\right]+[N, N]_{p} \\
& =\left[\cdots \left[[s(x), \underbrace{n], n], \cdots n}_{p}]+[N, N]_{p}\right.\right. \\
& =0
\end{aligned}
$$

It follows that $(-)^{[p]}: N_{a b} \rightarrow N_{a b}^{L}$ and $\left(N_{a b},(-)^{[p]}\right)$ is a $w(L)$-module.

Lemma 3.1. If $\phi: E \rightarrow L$ is a restricted Lie epimorphism with kernel $N$ and $(A, f)$ is a $w(L)$-module, then we have an isomorphism

$$
\operatorname{Tors}^{0}(\phi,(A, f)) \simeq \operatorname{Hom}_{w(L)}\left(\left(N_{a b},(-)^{[p]}\right),(A, f)\right)
$$

Proof. Let $E$. be the simplicial restricted Lie algebra which is obtained by iterating the simplicial kernel construction, i.e.,

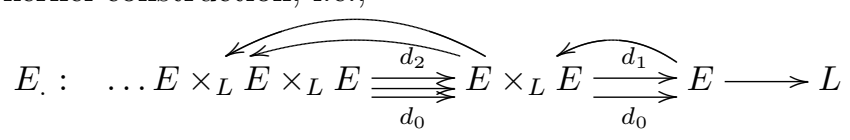

We have an isomorphism of restricted Lie algebras

$$
E \rtimes N \simeq E \times{ }_{L} E
$$

given by

$$
(x, n) \mapsto(x, x+n)
$$

Therefore we get the following simplicial object:

$$
E .: \ldots E \rtimes N \rtimes N \underset{\frac{d_{2}}{d_{d_{0}}}}{\xi} E \rtimes N \underset{\underset{d_{0}}{\stackrel{d_{1}}{\longrightarrow}}}{\longrightarrow} E \longrightarrow L
$$

where

$$
\begin{aligned}
d_{0}\left(x, n, n^{\prime}\right): & =(x, n) \\
d_{1}\left(x, n, n^{\prime}\right): & =\left(x, n^{\prime}\right) \\
d_{2}\left(x, n, n^{\prime}\right): & =\left(x+n, n^{\prime}-n\right)
\end{aligned}
$$

The abelian group $\operatorname{Tors}^{0}(\phi,(A, f))$ is defined by

$$
\operatorname{Tors}^{0}(\phi,(A, f)):=\operatorname{Hom}_{\operatorname{Simpl}(\mathrm{RLie} / \mathrm{L})}\left(E_{.}, K\left(L \times_{f} A, 1\right)\right)
$$

and by Lemma 2.1 in $[\mathbf{7}]$ we obtain

$$
\begin{aligned}
& \operatorname{Tors}^{0}(\phi,(A, f)) \\
& \quad=\operatorname{ker}\left(\operatorname{Hom}_{\mathrm{RLie} / L}\left(E \rtimes N, L \times_{f} A\right) \stackrel{d_{0}^{*}-d_{1}^{*}+d_{2}^{*}}{\longrightarrow} \operatorname{Hom}_{\mathrm{RLie} / L}\left(E \rtimes N \rtimes N, L \times_{f} A\right)\right)
\end{aligned}
$$

If $\tau \in \operatorname{Tors}^{0}(\phi,(A, f))$, then by isomorphism (4) any $\tau$ is associated to a Beck 
derivation $d_{\tau} \in \operatorname{Der}_{p}(E \rtimes N,(A, f))$ such that

$$
d_{\tau} d_{0}-d_{\tau} d_{1}+d_{\tau} d_{2}=0
$$

i.e.,

$$
d_{\tau}(x, n)-d_{\tau}\left(x, n^{\prime}\right)+d_{\tau}\left(x+n, n^{\prime}-n\right)=0
$$

for all $x \in E$ and $n, n^{\prime} \in N$. Since $N=\operatorname{ker} \phi$, we get

$$
\begin{aligned}
d_{\tau}\left(0,\left[n, n^{\prime}\right]\right) & =d_{\tau}\left(\left[(0, n),\left(0, n^{\prime}\right)\right]\right) \\
& =(0, n) d_{\tau}\left(\left(0, n^{\prime}\right)\right)-\left(0, n^{\prime}\right) d_{\tau}((0, n)) \\
& =0
\end{aligned}
$$

Besides,

$$
\begin{aligned}
d_{\tau}\left(0,\left[n, n^{\prime}\right]\right. & =d_{\tau}\left(\left(0,\left[n, n^{\prime}\right]\right)^{[p]}\right) \\
& =(0 \underbrace{\left.\left[n, n^{\prime}\right]\right) \cdots(0,}_{p-1}\left[n, n^{\prime}\right]) d_{\tau}\left(0,\left[n, n^{\prime}\right]\right)+f\left(d_{\tau}\left(0,\left[n, n^{\prime}\right]\right)\right) \\
& =0
\end{aligned}
$$

Therefore a map $\bar{\tau}: N_{a b} \rightarrow A$ is defined by

$$
\bar{\tau}\left(n+[N, N]_{p}\right):=d_{\tau}(0, n)
$$

for all $n \in N$. Moreover, for $x \in L$ and $n \in N$ we have

$$
\begin{aligned}
\bar{\tau}\left(x \cdot\left(n+[N, N]_{p}\right)\right) & =\bar{\tau}\left([s(x), n]+[N, N]_{p}\right) \\
& =d_{\tau}(0,[s(x), n]) \\
& =d_{\tau}([(s(x), 0),(0, n)]) \\
& =(s(x), 0) d_{\tau}(0, n)-(0, n) d_{\tau}(s(x), 0) \\
& =(s(x), 0) d_{\tau}(0, n) \\
& =x \cdot \bar{\tau}\left(n+[N, N]_{p}\right) \\
\bar{\tau}\left(n^{[p]}+[N, N]_{p}\right)= & d_{\tau}\left(0, n^{[p]}\right) \\
= & d_{\tau}\left((0, n)^{[p]}\right) \\
= & \underbrace{(0, n) \cdots(0, n)}_{p-1} d_{\tau}(0, n)+f\left(d_{\tau}(0, n)\right) \\
= & f\left(d_{\tau}(0, n)\right)
\end{aligned}
$$

It follows that $\bar{\tau}$ is a morphism of $w(L)$-modules. Conversely, let

$$
\bar{\tau}: \operatorname{Hom}_{w(L)}\left(\left(N_{a b},(-)^{[p]}\right),(A, f)\right)
$$

be a $w(L)$-homomorphism. We define $\tau: E \rtimes N \rightarrow L \times_{f} A$ given by

$$
\tau(x, n):=\left(\phi(x), \bar{\tau}\left(n+[N, N]_{p}\right)\right)
$$

Then the associated derivation $d_{\tau}(x, n):=\bar{\tau}\left(n+[N, N]_{p}\right)$ satisfies the relation (6) and it follows that $\tau \in \operatorname{Tors}^{0}(\phi,(A, f))$. If $\tau, \tau^{\prime} \in \operatorname{Tors}^{0}(\phi,(A, f))$, then by isomorphism 
(4) $\tau+\tau^{\prime}$ is associated to the Beck derivation $d_{\tau}+d_{\tau^{\prime}}$. Thus it follows by definition that $\overline{\tau+\tau^{\prime}}=\bar{\tau}+\bar{\tau}^{\prime}$. Therefore the map $\bar{\tau} \mapsto \tau$ is a well defined homomorphism, inverse to the homomorphism $\tau \mapsto \bar{\tau}$.

We recall from Theorem 2.5 in $[\mathbf{9}]$ that the set $\operatorname{Ext}_{p}^{1}(L,(A, f))$ of equivalent classes of abelian extensions of restricted Lie algebras

$$
0 \rightarrow A \rightarrow E \rightarrow L \rightarrow 0
$$

is in bijection with the first Quillen-Barr-Beck cohomology group $H_{\mathbf{G}}^{1}(L,(A, f))$. Thus an abelian group structure is induced on the set $\operatorname{Ext}_{p}^{1}(L,(A, f))$ and, by Theorem 2.13 , there is an isomorphism

$$
\operatorname{Ext}_{p}^{1}(L,(A, f)) \simeq \operatorname{Tors}^{1}(L,(A, f))
$$

Theorem 3.2. Let $0 \rightarrow N \rightarrow E \rightarrow L \rightarrow 0$ be an exact sequence of restricted Lie algebras and let $(A, f)$ be a $w(L)$-module. Then there are naturally equivalent exact sequences

$$
\begin{array}{r}
0 \rightarrow \operatorname{Der}_{p}(L,(A, f)) \rightarrow \operatorname{Der}_{p}(E,(A, f)) \rightarrow \operatorname{Hom}_{w(L)}\left(\left(N_{a b},(-)^{[p]}\right),(A, f)\right) \rightarrow \\
\rightarrow \operatorname{Ext}_{p}^{1}(L,(A, f)) \rightarrow \operatorname{Ext}_{p}^{1}(E,(A, f)) \\
\begin{aligned}
0 \rightarrow H_{\mathbf{G}}^{0}(L,(A, f)) \rightarrow H_{\mathbf{G}}^{0}(E,(A, f)) \rightarrow \operatorname{Hom}_{w(L)}\left(\left(N_{a b},(-)^{[p]}\right),(A, f)\right) \rightarrow \\
\rightarrow H_{\mathbf{G}}^{1}(L,(A, f)) \rightarrow H_{\mathbf{G}}^{1}(E,(A, f))
\end{aligned}
\end{array}
$$

Proof. Since RLie is a monadic category over Sets whose objects have an underlying group structure, the theorem follows from the above lemma and the exact sequence (5).

\section{Internal groupoids and crossed modules}

Crossed modules in groups were introduced by Whitehead [31] in the study of relative homotopy groups. Brown and Spencer in [5] noted that internal categories within the category of groups are equivalent to crossed modules. In the more general context of categories of groups with operations, crossed modules and internal categories are studied by Porter in [22]. Moreover, internal categories in a Mal'tsev variety are studied by Janelidze in [19]. Also, in [13], Ellis introduced and studied the non-abelian tensor product of Lie algebras. Below we consider the case of the category of restricted Lie algebras.

Let us recall the definition of an internal category in a category $\mathcal{C}$ with pullbacks. An internal category $C=\left(C_{0}, C_{1}, e, \mathfrak{s}, t, \theta\right)$ in $\mathcal{C}$ is a diagram in $\mathcal{C}$

$$
C_{1} \times{ }_{C_{0}} C_{1} \stackrel{\theta}{\longrightarrow} C_{1} \underset{t}{\stackrel{\stackrel{s}{\mathfrak{s}}}{\longrightarrow}} C_{0}
$$

such that $t e=\mathfrak{s} e=i d_{C_{0}}$ with a morphism $\theta: C_{1} \times_{C_{0}} C \rightarrow C_{1}$, where $C_{1} \times_{C_{0}} C_{1}$ 
denotes the pullback

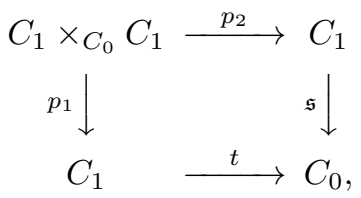

satisfying: $t \theta=t p_{2}$ and $\mathfrak{s} \theta=\mathfrak{s} p_{1}$, the associative law relation

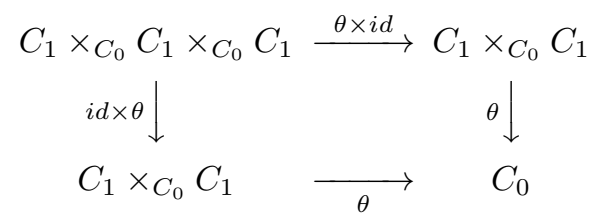

and the left and right unit laws for composition of morphisms

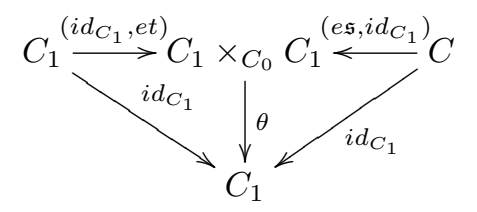

The morphisms $t, \mathfrak{s}, e$ are called the target, source, and unit morphisms respectively. An internal category is called internal groupoid if for any $c \in \mathcal{C}$ there is a $c^{\prime} \in \mathcal{C}$ such that $\theta\left(c, c^{\prime}\right)=e \mathfrak{s}(c)$ and $\theta\left(c^{\prime}, c\right)=e t(c)$.

An internal functor between two internal categories $C$ and $D$ in $\mathcal{C}$ is defined to be a pair of morphisms $f_{0}: C_{0} \rightarrow D_{0}$ and $f_{1}: C_{1} \rightarrow D_{1}$ of $\mathcal{C}$ such that the following diagrams are commutative:

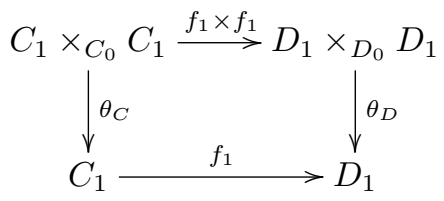

and

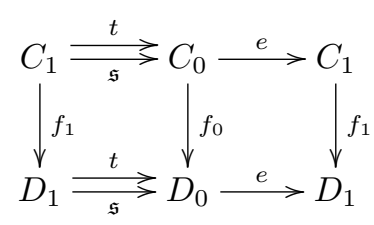

We denote by Grp the category whose objects are internal groupoids in RLie and whose morphisms are internal functors. J.-L. Loday and C. Kassel define in [20] the notion of crossed modules for the category of Lie algebras. In the same way crossed modules for the category of restricted Lie algebras are defined (cf. [8]).

Definition 4.1. Let $\mu: M \rightarrow N$ be a homomorphism of restricted Lie algebras. The triple $(M, N, \mu)$ is called a crossed module if there is given a restricted homomorphism $\eta: N \rightarrow \operatorname{Der}(M)$ such that $\eta(n)$ is a restricted derivation for all $n \in N$ and 
the following relations hold:

$$
\begin{aligned}
\mu(\eta(n)(m)) & =[n, \mu(m)], \quad n \in N, m \in M \\
\eta(\mu(m))\left(m^{\prime}\right) & =\left[m, m^{\prime}\right], \quad m, m^{\prime} \in M
\end{aligned}
$$

Example 4.2. Let $L$ be a restricted Lie algebra and $I$ an ideal of $L$. If $i: I \hookrightarrow L$ denotes the inclusion homomorphism, then the $(I, L, i)$ is a crossed module in RLie.

A morphism between two crossed modules $(M, N, \mu)$ and $\left(M^{\prime}, N^{\prime}, \mu\right)$ is defined to be a pair of restricted Lie homomorphisms $f_{1}: M \rightarrow M^{\prime}$ and $f_{0}: N \rightarrow N^{\prime}$ such that the following diagram is commutative:

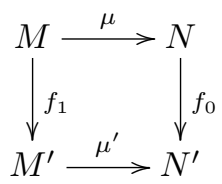

and

$$
f_{1}(\eta(n)(m))=\eta^{\prime}\left(f_{0}(n)\right)\left(f_{1}(m)\right), \text { for all } n \in N, m \in M
$$

The corresponding category of crossed modules in RLie is denoted by CM.

Theorem 4.3. The categories of crossed modules and internal groupoids in RLie are equivalent.

Proof. To any crossed module $(M, N, \mu)$ we associate the diagram in RLie

$$
(M \rtimes N) \times_{N}(M \rtimes N) \stackrel{\theta}{\longrightarrow}(M \rtimes N) \underset{t}{\stackrel{\stackrel{e}{\mathfrak{s}}}{\longrightarrow}} N
$$

where $\mathfrak{s}, t: M \rtimes N \rightarrow N$ are restricted Lie homomorphisms given by

$$
\begin{aligned}
\mathfrak{s}(m, n) & :=n, \\
t(m, n) & :=n+\mu(m),
\end{aligned}
$$

and $e: N \rightarrow M \rtimes N$ by

$$
e(n):=(0, n)
$$

for all $m \in M$ and $n \in N$. The multiplication

$$
\theta:(M \rtimes N) \times_{N}(M \rtimes N) \rightarrow M \rtimes N
$$

is given by

$$
\theta\left((m, n),\left(m^{\prime}, n+\mu(m)\right)\right)=\left(m+m^{\prime}, n\right)
$$

A straightforward calculation shows that $\theta$ is a Lie algebra homomorphism. Moreover, 
we note that

$$
\begin{aligned}
\theta\left((m, 0),(0, \mu(m))^{[p]}\right) & =\theta\left((m, 0)^{[p]},(0, \mu(m))^{[p]}\right) \\
& =\theta\left(\left(m^{[p]}, 0\right),\left(0, \mu(m)^{[p]}\right)\right) \\
& =\theta\left(\left(m^{[p]}, 0\right),\left(0, \mu\left(m^{[p]}\right)\right)\right) \\
& =\left(m^{[p]}, 0\right) \\
& =(\theta((m, 0),(0, \mu(m))))^{[p]}
\end{aligned}
$$

and in the same way we see that

$$
\theta\left(((0, n),(0, n))^{[p]}\right)=(\theta((0, n),(0, n)))^{[p]}
$$

and

$$
\theta\left(\left((0,0),\left(m^{\prime}, 0\right)\right)^{[p]}\right)=\left(\theta\left((0,0),\left(m^{\prime}, 0\right)\right)\right)^{[p]}
$$

Since $\theta$ is a Lie algebra homomorphism, we deduce that $\theta$ is actually a restricted Lie homomorphism. Then one can easily check that the conditions of associativity and unit and right laws are satisfied. Besides, if we define

$$
(m, n)^{\prime}:=(-m, n+\mu(m))
$$

for all $m \in M$ and $n \in N$, then we see that the above diagram in RLie defines an internal groupoid in RLie.

If

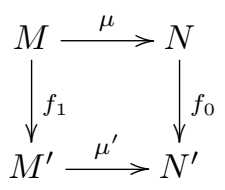

is a morphism of crossed modules, then we see that the following diagram is commutative

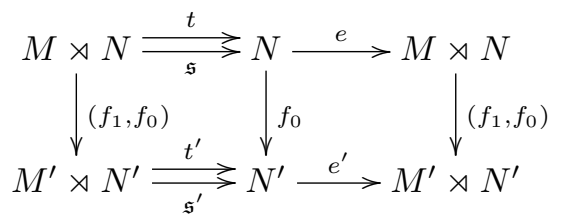

For $m_{1}, m_{2} \in M$ and $n \in N$ we have

$$
\begin{aligned}
\left(f_{1}, f_{0}\right) \theta\left(\left(m_{1}, n\right),\left(m_{2}, n+\mu\left(m_{1}\right)\right)\right) & =\left(f_{1}, f_{0}\right)\left(m_{1}+m_{2}, n\right) \\
& =\left(f_{1}\left(m_{1}+m_{2}\right), f_{0}(n)\right) \\
& =\left(f_{1}\left(m_{1}\right)+f_{1}\left(m_{2}\right), f_{0}(n)\right)
\end{aligned}
$$


and

$$
\begin{aligned}
\theta^{\prime}\left(\left(f_{1}, f_{0}\right) \times\left(f_{1}, f_{0}\right)\right)\left(\left(m_{1}, n\right),\right. & \left.\left(m_{2}, n+\mu\left(m_{1}\right)\right)\right)= \\
= & \theta^{\prime}\left(\left(f_{1}\left(m_{1}\right), f_{0}(n)\right),\left(f_{1}\left(m_{2}\right), f_{0}\left(n+\mu\left(m_{1}\right)\right)\right)\right) \\
= & \theta^{\prime}\left(\left(f_{1}\left(m_{1}\right), f_{0}(n)\right),\left(f_{1}\left(m_{2}\right), f_{0}(n)+\mu^{\prime} f_{1}\left(m_{1}\right)\right)\right) \\
= & \left(f_{1}\left(m_{1}\right)+f_{1}\left(m_{2}\right), f_{0}(n)\right)
\end{aligned}
$$

Therefore the following diagram is commutative:

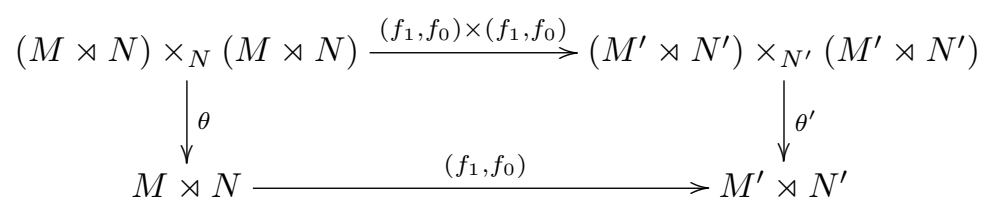

In this way a functor $F: \mathrm{CM} \rightarrow$ Grp is constructed. To go in the other direction, let

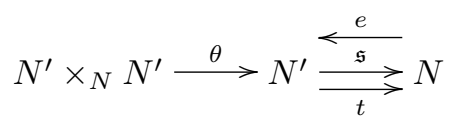

be an internal groupoid in RLie with multiplication $\theta$. Then we associate to it a crossed module $(M, N, \mu)$ where $M:=\operatorname{Ker} \mathfrak{s}$ and $\mu:=\left.t\right|_{M}$ and with action $\eta: N \rightarrow \operatorname{Der}(M)$ given by

$$
\eta(n)(m)=[e(n), m]
$$

for all $n \in N$ and $m \in M$. In effect,

$$
\begin{aligned}
\eta\left(n^{[p]}\right)(m) & =\left[e\left(n^{[p]}\right), m\right] \\
& =\left[(e(n))^{[p]}, m\right] \\
& =(\eta(n))^{p}(m)
\end{aligned}
$$

and

$$
\begin{aligned}
\eta(n)\left(m^{[p]}\right) & =\left[e(n), m^{[p]}\right] \\
& =a d_{m}^{p-1}(\eta(n)(m))
\end{aligned}
$$

Since $t e=\mathfrak{s} e=\left.i d\right|_{N}$, we get

$$
\theta\left(m+e(n), m^{\prime}+e(n+\mu(m))\right)=\theta(m+e(n), e t(m+e(n)))+\theta\left(e \mathfrak{s}\left(m^{\prime}\right), m^{\prime}\right)
$$

Besides, by unities properties of the groupoid we have that

$$
\theta(m+e(n), e t(m+e(n)))+\theta\left(e \mathfrak{s}\left(m^{\prime}\right), m^{\prime}\right)=m+e(n)+m^{\prime}
$$

Thus we obtain

$$
\theta\left(m+e(n), m^{\prime}+e(n+\mu(m))\right)=m+m^{\prime}+e(n)
$$

Since $\theta$ is a Lie algebra homomorphism, we have

$$
\theta\left(\left[\left(m, m^{\prime}+e \mu(m)\right),\left(0, m^{\prime \prime}\right)\right]\right)=\left[\theta\left(m, m^{\prime}+e \mu(m)\right), \theta\left(0, m^{\prime \prime}\right)\right]
$$


and

$$
\left[e \mu(m), m^{\prime \prime}\right]+\left[m^{\prime}, m^{\prime \prime}\right]=\left[m, m^{\prime \prime}\right]+\left[m^{\prime}, m^{\prime \prime}\right]
$$

We deduce that

$$
\begin{aligned}
\eta(\mu(m))\left(m^{\prime \prime}\right) & =\left[e(\mu(m)), m^{\prime \prime}\right] \\
& =\left[m, m^{\prime \prime}\right]
\end{aligned}
$$

Moreover, we have

$$
\begin{aligned}
\mu(\eta(n)(m)) & =t([e(n), m]) \\
& =[t e(n), t(m)] \\
& =[n, \mu(m)]
\end{aligned}
$$

Let $\left(N, N^{\prime}, e, \mathfrak{s}, t, \theta\right)$ and $\left(L, L^{\prime}, e^{\prime}, \mathfrak{s}^{\prime}, t^{\prime}, \theta^{\prime}\right)$ be two groupoids with associated crossed modules $(M, N, \mu)$ and $\left(M^{\prime}, L, \mu^{\prime}\right)$ respectively. If a pair of morphisms $\left(f_{0}, f_{1}\right)$ defines an internal functor between the groupoids $\left(N, N^{\prime}, e, \mathfrak{s}, t, \theta\right)$ and $\left(L, L^{\prime}, e^{\prime}, \mathfrak{s}^{\prime}, t^{\prime}, \theta^{\prime}\right)$, then the following diagram is commutative:

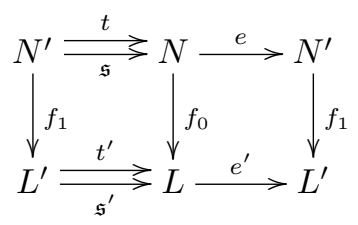

For $n \in N$ and $m \in M$ we have

$$
\begin{aligned}
\left.f_{1}\right|_{\text {Ker } \mathfrak{s}}([e(n), m]) & =\left[\left.f_{1}\right|_{K e r \mathfrak{s}}(e(n)),\left.f_{1}\right|_{\text {Ker } \mathfrak{s}}(m)\right] \\
& =\left[e^{\prime} f_{0}(n),\left.f_{1}\right|_{K e r \mathfrak{s}}(m)\right]
\end{aligned}
$$

Hence, the diagram below is a morphism of crossed modules:

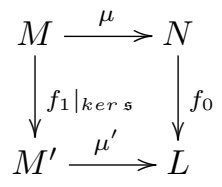

Therefore a functor $G: \operatorname{Grp} \rightarrow \mathrm{CM}$ is defined which is a quasi-inverse for $F$.

\section{The second cohomology group and 2-fold extensions}

Gerstenhaber in [14] studies 2-fold extensions in certain categories of interest including the category of Lie algebras. Also, the case of Lie algebras is studied by Shimada-Uehara-Brenneman-Iwai in [27]. Besides, in [20] J.-L. Loday and C. Kassel consider 2-fold extensions of Lie algebras associated to a crossed module. In this section we study 2 -fold extensions in the category of restricted Lie algebras.

Let $(F, E, \mu)$ be a crossed module in RLie. If $\left(A,(-)^{\left[p_{A}\right]}\right):=k e r \mu$, then by (8) we get that

$$
\mu(a) \cdot x=[a, x]=0
$$

for all $a \in A$ and $x \in F$. Thus, $A$ is contained in the center of $F$. Moreover, from (7), 
we have that

$$
\mu(e \cdot a)=[e, \mu(a)]=0
$$

thus $e \cdot a \in A$ for all $e \in E$ and $a \in A$.

The restriction on $A$ of the action of $E$ on $F$ endows $A$ with the structure of a restricted $E$-module. Since $\eta(e)$ is a restricted derivation for all $e \in E$, we have

$$
\begin{aligned}
e \cdot a^{\left[p_{A}\right]} & =a d_{a}^{p-1}(e \cdot a) \\
& =0
\end{aligned}
$$

Therefore the pair $\left(A,(-)^{\left[p_{A}\right]}\right)$ is a $w(E)$-module. Besides, $\mu(e \cdot x)=[e, \mu(x)]$ for all $e \in E$ and $x \in F$; thus we obtain that $\operatorname{Img}(\mu)$ is a restricted ideal of $E$. Since

$$
\begin{aligned}
(e+\mu(x)) \cdot a & =e \cdot a+[x, a] \\
& =e \cdot a+0
\end{aligned}
$$

one sees that $A$ is also a restricted $L$-module where $L:=\operatorname{coker} \mu$. Therefore $\left(A,\left(_{-}\right)^{\left[p_{A}\right]}\right)$ becomes a $w(L)$-module.

\subsection{2-fold extensions}

Let $(A, f)$ be a $w(L)$-module. We denote by $E^{2}(L,(A, f))$ the category whose objects are exact sequences in RLie

$$
0 \rightarrow A \stackrel{i}{\rightarrow} F \stackrel{\mu}{\rightarrow} E \stackrel{\phi}{\rightarrow} L \rightarrow 0
$$

where $(F, E, \mu)$ is a crossed module, such that the induced $w(L)$-module structure on $A$ is the given one. This implies that $f$ is the $p$-map of the restricted Lie algebra $A$. The morphisms are commutative diagrams of the form

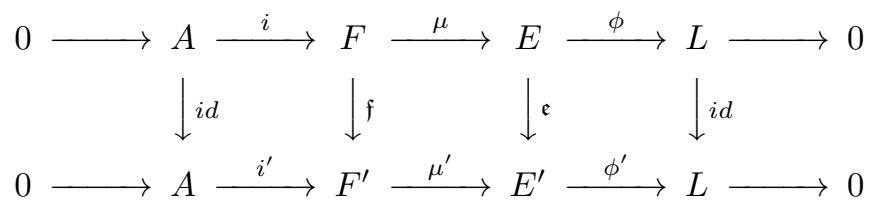

where the morphisms $\mathfrak{f}$ and $\mathfrak{e}$ respect the actions. Two objects $E_{1}, E_{2} \in E^{2}(L,(A, f))$ are called elementary equivalent if there is a morphism in $E^{2}(L,(A, f))$ from one to the other. We consider the equivalence relation generated by elementary equivalence. We denote by $\operatorname{Ext}_{p}^{2}(L,(A, f))$ the set of equivalence classes in $E^{2}(L,(A, f))$.

For $\phi: E \rightarrow L$, a fixed epimorphism in RLie, we consider the sub-category $E^{2}(\phi,(A, f))$ of $E^{2}(L,(A, f))$ whose objects are 2-fold extensions

$$
0 \rightarrow A \stackrel{i}{\rightarrow} F \stackrel{\mu}{\rightarrow} E \stackrel{\phi}{\rightarrow} R \rightarrow 0
$$

and morphisms

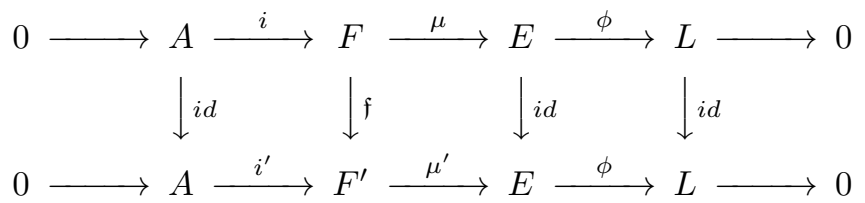

We denote by $\operatorname{Ext}_{p}^{2}(\phi,(A, f))$ the set of equivalence classes in $E^{2}(\phi,(A, f))$. 
Next we give an interpretation of 2-torsors over a restricted Lie algebra $L$ in terms of 2-fold extensions. Let $\phi: E_{0} \stackrel{\phi}{\rightarrow} L$ be a restricted Lie epimorphism and $(A, f) \in$ $a b(\mathrm{RLie} / L)$. Following $[\boldsymbol{7}]$, we define the category $\operatorname{TORS}(\phi,(A, f))$ whose objects are 2 -torsors over $L$ with fixed augmentation $\phi$ and whose morphisms are torsor maps $f .: E$. $\rightarrow E^{\prime}$. with $f_{0}=i d_{E_{0}}$. The corresponding category of connected components is denoted by $\operatorname{Tors}^{1}(\phi,(A, f))$.

Lemma 5.1. Let $L \in$ RLie be a restricted Lie algebra and $(A, f)$ a $w(L)$-module. Then we have bijections

$$
\operatorname{Tors}^{2}(L,(A, f)) \simeq \operatorname{Ext}_{p}^{2}(L,(A, f))
$$

and

$$
\operatorname{Tors}^{1}(\phi,(A, f)) \simeq \operatorname{Ext}_{p}^{2}(\phi,(A, f))
$$

Proof. Let $(E ., \epsilon)$ be a 2-torsor over $L$ under $(A, f)$, with augmentation $\phi: E_{0} \rightarrow L$. We consider the Moore complex $M\left(E\right.$.) of $(E$. $)$, which is given by $M(E .)_{0}=E_{0}$ and, for $n \geqslant 1$, by

$$
M(E .)_{n}=\cap_{i}^{n} \operatorname{ker} d_{i}=\operatorname{ker}\left(E_{n} \rightarrow \Lambda^{0}(n-1)(E .)\right)
$$

and whose differential is defined by restriction of the 0 -face operator of $E$. Therefore by conditions 1 and 3 of Definition 2.11 the associated Moore complex $M(E$.) is given by the following exact sequence in RLie:

$$
M(E .): 0 \rightarrow K_{\text {er }} d_{1} \cap \operatorname{ker} d_{2} \stackrel{d_{0}}{\longrightarrow} \operatorname{ker} d_{1} \stackrel{d_{0}}{\longrightarrow} E_{0} \stackrel{\phi}{\rightarrow} L \rightarrow 0
$$

It follows from condition 2 of Definition 2.11 that we have a pullback diagram

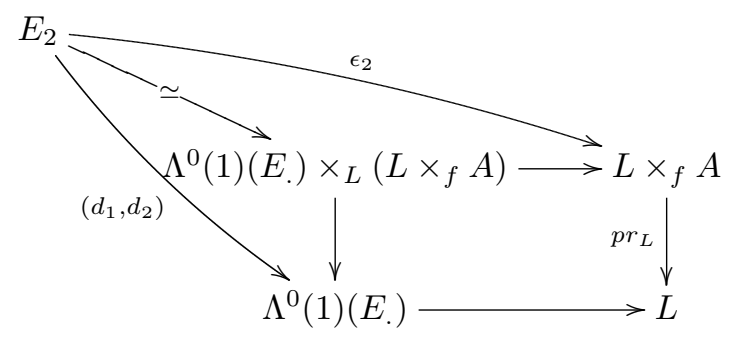

where

$$
\Lambda^{0}(1)\left(E_{.}\right)=\left\{\left(e_{1}, e_{1}^{\prime}\right), \mid d_{1}\left(e_{1}\right)=d_{1}\left(e_{1}^{\prime}\right), e_{1}, e_{1}^{\prime} \in E_{1}\right\}
$$

and

$$
E_{2} \simeq \Lambda^{0}(1)(E .) \times_{L}\left(L \times_{f} A\right)
$$

The restriction of $\epsilon_{2}$ on $\operatorname{Ker} d_{1} \cap$ ker $d_{2}$ induces an isomorphism

$$
\overline{\epsilon_{2}}: \operatorname{Ker} d_{1} \cap \operatorname{ker} d_{2} \stackrel{\sim}{\rightarrow} A
$$

Thus we obtain an exact sequence in RLie

$$
0 \rightarrow A \stackrel{d_{0} \bar{\epsilon}^{-1}}{\longrightarrow} \operatorname{Kerd}_{1} \stackrel{d_{0}}{\longrightarrow} E_{0} \stackrel{\phi}{\rightarrow} L \rightarrow 0
$$


The simplicial truncated diagram

$$
E_{1} \underset{d_{0}}{\stackrel{\stackrel{s_{0}}{d_{1}}}{\longrightarrow}} E_{0}
$$

defines an internal groupoid. In particular, $E_{1} \simeq \operatorname{Ker} d_{1} \rtimes E_{0}$ and the multiplication is necessarily given by

$$
\theta\left(x+s_{0}\left(e_{0}\right), x^{\prime}+s_{0}\left(d_{0}(x)+e_{0}\right)\right)=x+s_{0}\left(e_{0}\right)+x^{\prime}
$$

since

$$
\begin{aligned}
\theta\left(x+s_{0}\left(e_{0}\right), x^{\prime}+s_{0}\left(d_{0}(x)+e_{0}\right)\right) & =\theta\left(x+s_{0}\left(e_{0}\right), s_{0} d_{0}(x)+s_{0}\left(e_{0}\right)\right)+\theta\left(0, x^{\prime}\right) \\
& =\theta\left(i d, s_{0} d_{0}\right)\left(x+s_{0}\left(e_{0}\right)\right)+\theta\left(s_{0} d_{1}, i d\right)\left(x^{\prime}\right) \\
& =x+s_{0}\left(e_{0}\right)+x^{\prime}
\end{aligned}
$$

for all $x, x^{\prime} \in \operatorname{Ker} d_{1}$ and $e_{0} \in E_{0}$. By Theorem 4.3 it follows that $k e r d_{1} \stackrel{d_{0}}{\longrightarrow} E_{0}$ is a crossed module in RLie associated to the above internal groupoid.

Next we prove that the Lie action of $L$ on $A$ coincides with the Lie action induced from the exact sequence (9) (cf. $[\mathbf{2 9}])$. Let $\Lambda^{2}(1)(E$.) be the 2-horn

$$
\Lambda^{2}(1)(E .):=\left\{\left(e_{1}, e_{1}^{\prime}\right), \mid d_{0}\left(e_{1}\right)=d_{0}\left(e_{1}^{\prime}\right), e_{1}, e_{1}^{\prime}, E_{1}\right\}
$$

We denote by $p r: \Lambda^{2}(1)(E$. $) \rightarrow E_{1}$ the projection given by $\operatorname{pr}\left(e_{1}, e_{2}\right):=e_{1}$. By Definition 2.11 and its condition 2 the following diagram is a pullback:

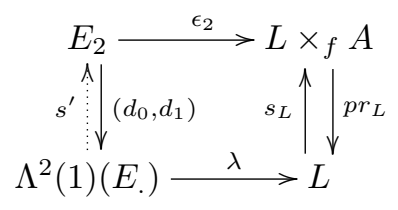

where $\lambda:=\phi d_{0} p r$. Therefore there is a section $s^{\prime}: \Lambda^{2}(1)(E.) \rightarrow E_{2}$ such that $\left(d_{0}, d_{1}\right) s^{\prime}$ $=i d$ and $\epsilon_{2} s^{\prime}=s_{L} \lambda$, where $s_{L}: L \rightarrow L \times_{f} A$ is given by $s_{L}(x)=(x, 0)$ for all $x \in L$. If $x \in L$ and $e_{0} \in E_{0}$ such that $\phi\left(e_{0}\right)=x$, then

$$
\begin{aligned}
d_{0}{\overline{\epsilon_{2}}}^{-1}(0, x \cdot a) & =d_{0}{\overline{\epsilon_{2}}}^{-1}\left(\left[s_{L} \phi\left(e_{0}\right),(0, a)\right]\right) \\
& =d_{0} \bar{\epsilon}^{-1}\left(\left[s_{L} \phi d_{0} p r\left(i d, s_{0} d_{0}\right) s_{0}\left(e_{0}\right),(0, a)\right]\right)
\end{aligned}
$$

where $\left(i d, s_{0} d_{0}\right): E_{1} \rightarrow \Lambda^{2}(1)(E$. $)$ is given by

$$
\left(i d, s_{0} d_{0}\right)\left(e_{1}\right):=\left(e_{1}, s_{0} d_{0}\left(e_{1}\right)\right)
$$

for all $e_{1} \in E_{1}$. Besides,

$$
\begin{aligned}
\epsilon_{2}\left(\left[s^{\prime}\left(i d, s_{0} d_{0}\right) s_{0}\left(e_{0}\right),{\overline{\epsilon_{2}}}^{-1}(0, a)\right]\right) & =\left[\epsilon_{2} s^{\prime}\left(i d, s_{0} d_{0}\right) s_{0}\left(e_{0}\right),(0, a)\right] \\
& =\left[s_{L} \phi d_{0} p r\left(i d, s_{0} d_{0}\right) s_{0}\left(e_{0}\right),(0, a)\right]
\end{aligned}
$$

Therefore,

$$
\begin{aligned}
d_{0}{\overline{\epsilon_{2}}}^{-1}(0, x \cdot a) & =d_{0}\left(\left[s^{\prime}\left(i d, s_{0} d_{0}\right) s_{0}\left(e_{0}\right), \epsilon_{2}^{-1}(0, a)\right]\right) \\
& =\left(\left[d_{0} s^{\prime}\left(i d, s_{0} d_{0}\right) s_{0}\left(e_{0}\right), d_{0}{\overline{\epsilon_{2}}}^{-1}(0, a)\right]\right)
\end{aligned}
$$


Since $\left(d_{0}, d_{1}\right) s^{\prime}=i d$, we get $d_{0} s^{\prime}\left(i d, s_{0} d_{0}\right) s_{0}\left(e_{0}\right)=s_{0}\left(e_{0}\right)$ and

$$
\begin{aligned}
d_{0} e_{2}^{-1}(0, x \cdot a) & =\left(\left[d_{0} s^{\prime}\left(i d, s_{0} d_{0}\right) s_{0}\left(e_{0}\right), d_{0}{\overline{\epsilon_{2}}}^{-1}(0, a)\right]\right) \\
& =\left[s_{0}\left(e_{0}\right), d_{0}{\overline{\epsilon_{2}}}^{-1}(0, a)\right]
\end{aligned}
$$

Moreover, we have

$$
\begin{aligned}
d_{0}{\overline{\epsilon_{2}}}^{-1}\left(0, x^{[p]} \cdot a\right) & =\left[s_{0}\left(e_{0}^{[p]}\right), d_{0}{\overline{\epsilon_{2}}}^{-1}(0, a)\right] \\
& =\left[\left(s_{0}\left(e_{0}\right)^{[p]}, a\right]\right. \\
& \left.\left.\left.=[\underbrace{s_{0}\left(e_{0}\right),\left[s_{0}\left(e_{0}\right),\left[\cdots \left[s_{0}\left(e_{0}\right)\right.\right.\right.}_{p}, d_{0}{\overline{\epsilon_{2}}}^{-1}(0, a)]\right]\right]\right]
\end{aligned}
$$

Therefore the induced $w(L)$-module structure by the 2 -fold exact sequence coincides with the initial structure and we obtain an extension in $E^{2}(L,(A, f))$

$$
0 \rightarrow A \stackrel{d_{0} \bar{\epsilon}^{-1}}{\longrightarrow} \operatorname{Kerd}_{1} \stackrel{d_{0}}{\longrightarrow} E_{0} \stackrel{\phi}{\rightarrow} L \rightarrow 0
$$

Notice that a 2 -torsor over $L$ with augmentation $\phi$ is associated to a 2 -fold extension in $E^{2}(\phi,(A, f))$. Besides, a morphism of 2 torsors

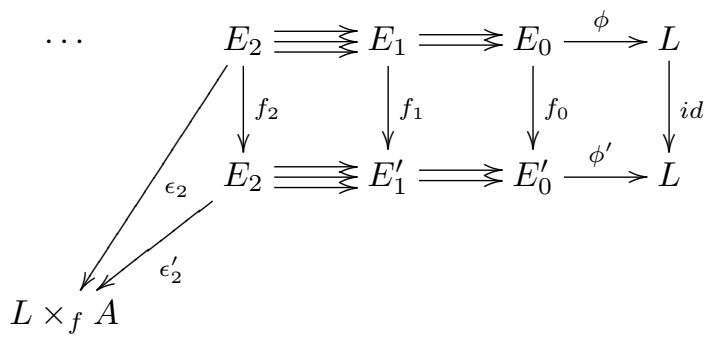

induces a morphism of 2 -fold extensions

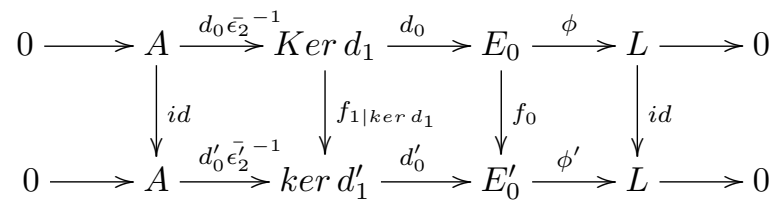

Thus are induced maps

$$
\psi: \operatorname{Tors}^{2}(L,(A, f)) \rightarrow \operatorname{Ext}_{p}^{2}(L,(A, f))
$$

and

$$
\psi^{\prime}: \operatorname{Tors}^{2}(\phi,(A, f)) \rightarrow \operatorname{Ext}_{p}^{2}(\phi,(A, f))
$$

Conversely, let

$$
0 \rightarrow A \stackrel{i}{\rightarrow} F \stackrel{\mu}{\rightarrow} E_{0} \stackrel{\phi}{\rightarrow} L \rightarrow 0
$$

be a 2-fold extension in $E^{2}(L,(A, f))$. By Theorem 4.3 is associated an augmented 
over $L$ internal groupoid $\Gamma$ :

$$
E_{1}:=\left(E_{0} \rtimes F\right) \underset{d_{1}}{\stackrel{<d_{0}}{\longrightarrow}} E_{0} \stackrel{\phi}{\longrightarrow} L \longrightarrow 0
$$

where $d_{1}\left(e_{0}, x\right):=e_{0}, d_{0}\left(e_{0}, x\right):=\mu(x)+e_{0}$ and $s_{0}\left(e_{0}\right):=\left(e_{0}, 0\right)$ for all $e_{0} \in E_{0}$ and $x \in F$. Let $E$. $:=\operatorname{cosk}^{1}(\Gamma)$ be the 1-coskeleton of $\Gamma$. If

$$
\left(\left(\mu(z)+e_{0}, x\right),\left(e_{0}, y\right),\left(e_{0}, z\right)\right) \in E_{2}
$$

where $x, y, z \in F$ and $e_{0} \in E_{0}$, then $x+z-y \in A$.

We define a linear map $\epsilon_{2}: E_{2} \rightarrow L \times_{f} A$ given by

$$
\epsilon_{2}\left(\left(\mu(z)+e_{0}, x\right),\left(e_{0}, y\right),\left(e_{0}, z\right)\right)=\left(\phi\left(e_{0}\right), x+z-y\right)
$$

Then $\epsilon_{2}$ is a Lie algebra homomorphism since

$$
\begin{aligned}
\epsilon_{2}([((\mu(z), x), & \left.\left.(0, y),(0, z)),\left(\left(\mu\left(z^{\prime}\right), x^{\prime}\right),\left(0, y^{\prime}\right),\left(0, z^{\prime}\right)\right)\right]\right)= \\
& =\epsilon_{2}\left(\left(\mu\left(\left[z, z^{\prime}\right]\right),\left[z, x^{\prime}\right]-\left[z^{\prime}, x\right]+\left[x, x^{\prime}\right]\right),\left(0,\left[y, y^{\prime}\right]\right),\left(0,\left[z, z^{\prime}\right]\right)\right) \\
& =\left(\phi(0),\left[y-x, x^{\prime}\right]-\left[y^{\prime}-x^{\prime}, x\right]+\left[x, x^{\prime}\right]+\left[y-x, y^{\prime}-x^{\prime}\right]-\left[y, y^{\prime}\right]\right) \\
& =(0,0)
\end{aligned}
$$

and

$$
\begin{aligned}
{\left[\epsilon_{2}((\mu(z), x),(0, y),(0, z)), \epsilon_{2}\left(\left(\mu\left(z^{\prime}\right), x^{\prime}\right),\right.\right.} & \left.\left.\left(0, y^{\prime}\right),\left(0, z^{\prime}\right)\right)\right]= \\
& =\left[(0, x+z-y),\left(0, x^{\prime}+z^{\prime}-y^{\prime}\right)\right] \\
& =\left(0,\left[x+z-y, x^{\prime}+z^{\prime}-y^{\prime}\right]\right)=(0,0)
\end{aligned}
$$

Besides,

$$
\begin{aligned}
\epsilon_{2}([((\mu(z), x),(0, y),(0, z)), & \left.\left.\left(\left(e_{0}, 0\right),\left(e_{0}, 0\right),\left(e_{0}, 0\right)\right)\right]\right)= \\
& \left.=\epsilon_{2}\left(\left[\mu(z), e_{0}\right],-e_{0} \cdot x\right),\left(0,-e_{0} \cdot y\right),\left(0,-e_{0} \cdot z\right)\right) \\
& =\epsilon_{2}\left(\left(-\mu\left(e_{0} \cdot z\right),-e_{0} \cdot x\right),\left(0,-e_{0} \cdot y\right),\left(0,-e_{0} \cdot z\right)\right) \\
& =\left(0,-e_{0} \cdot x+e_{0} \cdot y,-e_{0} \cdot z\right)
\end{aligned}
$$

and

$$
\begin{aligned}
{\left[\epsilon_{2}((\mu(z), x),(0, y),(0, z)), \epsilon_{2}\left(\left(e_{0}, 0\right),\left(e_{0}, 0\right),\left(e_{0}, 0\right)\right)\right] } & =\left[(0, x+z-y),\left(\phi\left(e_{0}\right), 0\right)\right] \\
& =\left(0,-e_{0} \cdot(x+z-y)\right)
\end{aligned}
$$

Obviously,

$$
\begin{aligned}
\epsilon_{2}\left(\left[\left(\left(e_{0}, 0\right),\left(e_{0}, 0\right),\left(e_{0}, 0\right)\right),\right.\right. & \left.\left.\left(\left(e_{0}^{\prime}, 0\right),\left(e_{0}^{\prime}, 0\right),\left(e_{0}^{\prime}, 0\right)\right)\right]\right)= \\
& =\left[\epsilon_{2}\left(\left(e_{0}, 0\right),\left(e_{0}, 0\right),\left(e_{0}, 0\right)\right), \epsilon_{2}\left(\left(e_{0}^{\prime}, 0\right),\left(e_{0}^{\prime}, 0\right),\left(e_{0}^{\prime}, 0\right)\right)\right] \\
& =\left(\left[\phi\left(e_{0}\right), \phi\left(e_{0}^{\prime}\right)\right], 0\right)
\end{aligned}
$$

Since $z+x=y+a$ for some $a \in A$, the Lie algebra $L_{(z+x), y}$ generated by $(z+x)$ 
and $y$ is zero. By Remark 2.2 we obtain

$$
\begin{aligned}
(z+x-y)^{[p]} & =(z+x)^{[p]}-y^{[p]}+\sum_{i=1}^{i=p} s_{i}((z+x), y) \\
& =(z+x)^{[p]}-y^{[p]} \\
& =z^{[p]}+x^{[p]}+\sum_{i=0}^{i=1} s_{i}(z, x)-y^{[p]}
\end{aligned}
$$

By relation (8) we have $\mu(z) \cdot x=[z, x]$; thus

$$
\begin{aligned}
\epsilon_{2}\left(((\mu(z), x),(0, y),(0, z))^{[p]}\right)= & \epsilon_{2}\left(\left((\mu(z), x)^{[p]},(0, y)^{[p]},(0, z)^{[p]}\right)\right) \\
= & \epsilon_{2}\left(\left(\left(\mu(z)^{[p]}, 0\right)+\left(0, x^{[p]}\right)\right.\right. \\
& \left.\left.+\sum_{i=1}^{i=p} s_{i}((\mu(z), 0),(0, x))\right),\left(0, y^{[p]}\right),\left(0, z^{[p]}\right)\right) \\
= & \epsilon_{2}\left(\left(\mu\left(z^{[p]}\right), x^{[p]}+\sum_{i=1}^{i=p} s_{i}(z, x)\right),\left(0, y^{[p]}\right),\left(0, z^{[p]}\right)\right) \\
= & \left(0, x^{[p]}+\sum_{i=1}^{i=p} s_{i}(z, x)+z^{[p]}-y^{[p]}\right) \\
= & \epsilon_{2}((\mu(z), x),(0, y),(0, z))^{[p]}
\end{aligned}
$$

Also, $\phi$ is a restricted Lie homomorphism so

$$
\epsilon_{2}\left(\left(\left(e_{0}, 0\right),\left(e_{0}, 0\right),\left(e_{0}, 0\right)\right)^{[p]}\right)=\epsilon_{2}\left(\left(e_{0}, 0\right),\left(e_{0}, 0\right),\left(e_{0}, 0\right)\right)^{[p]}
$$

Since $\epsilon_{2}$ is a Lie algebra homomorphism, we see that $\epsilon_{2}$ is actually a restricted Lie homomorphism. Besides, $\epsilon_{2}$ is a cocycle, i.e.,

$$
\epsilon_{2}\left(d_{0}-d_{1}+d_{2}-d_{3}\right)=0
$$

In particular, let $\kappa=\left(t_{0}, t_{1}, t_{2}, t_{3}\right) \in E_{3}$ where

$$
\begin{aligned}
& t_{0}:=\left(\left(\mu(z)+e_{0}, y-z+a_{1}\right),\left(\mu(w)+e_{0}, y-w+a_{3}\right),\left(\mu(w)+e_{0}, z-w+a_{2}\right)\right) \\
& t_{1}:=\left(\left(\mu(z)+e_{0}, y-z+a_{1}\right),\left(e_{0}, y\right),\left(e_{0}, z\right)\right) \\
& t_{2}:=\left(\left(\mu(w)+e_{0}, y-w+a_{3}\right),\left(e_{0}, y\right),\left(e_{0}, w\right)\right) \\
& t_{3}:=\left(\left(\mu(w)+e_{0}, z-w+a_{2}\right),\left(e_{0}, z\right),\left(e_{0}, w\right)\right)
\end{aligned}
$$

Then using the addition of the group structure of $L \times_{f} A$ we get

$$
\epsilon_{2}\left(d_{0}-d_{1}+d_{2}-d_{3}\right)(\kappa)=\left(\phi\left(e_{0}\right), a_{1}+a_{2}-a_{3}-a_{1}+a_{3}-a_{2}\right)=\left(\phi\left(e_{0}\right), 0\right)
$$

Moreover, $\epsilon_{2}$ is a normalized cocycle, i.e.,

$$
\epsilon_{2} s_{0}=\epsilon_{2} s_{1}=0
$$


Precisely, let $\left(e_{0}, x\right) \in E_{1}$; then

$$
s_{0}\left(\left(e_{0}, x\right)\right)=\left(\left(e_{0}, x\right),\left(e_{0}, x\right),\left(e_{0}, 0\right)\right)
$$

and

$$
\epsilon_{2}\left(s_{0}\left(e_{0}, x\right)\right)=\left(\phi\left(e_{0}\right), x+0-x\right)=\left(\phi\left(e_{0}\right), 0\right)
$$

Besides,

$$
s_{1}\left(\left(e_{0}, x\right)\right)=\left(\left(\mu(x)+e_{0}, 0\right),\left(e_{0}, x\right),\left(e_{0}, x\right)\right)
$$

and

$$
\epsilon_{2}\left(s_{1}\left(e_{0}, x\right)\right)=\left(\phi\left(e_{0}\right), 0+x-x\right)=\left(\phi\left(e_{0}\right), 0\right)
$$

Let $\sigma$ be a section of the cokernel map of $i: A \rightarrow F$ and $s$ a section of $\phi$. We consider the map $s_{1}: E_{0} \rightarrow E_{1}$ given by $s_{1}\left(e_{0}\right):=\left(e_{0}, \sigma\left(s \phi\left(e_{0}\right)-e_{0}\right)\right)$. The maps $s_{1}, s$ define a $U$-splitting on the truncated complex

$$
E_{1} \underset{d_{1}}{\stackrel{\stackrel{s_{0}}{d_{0}} \longrightarrow}{\longrightarrow}} E_{0} \stackrel{\phi}{\longrightarrow} L \longrightarrow 0
$$

and a $U$-splitting is induced on the coskeleton $E$.

If

$$
K_{1} \underset{p r_{1}}{\stackrel{p r_{0}}{\longrightarrow}} E_{0} \stackrel{\phi}{\longrightarrow} L \longrightarrow 0
$$

is the simplicial kernel of $E_{0} \rightarrow L$, then

$$
K_{1}:=\left\{\left(\mu(z)+e_{0}, e_{0}\right): e_{0} \in E_{0}, z \in F\right\}
$$

The 2-th component of the $U$-split $s_{2}: E_{1} \rightarrow E_{2}$ is given by

$$
s_{2}:=\left(s_{1} d_{0}, s_{1} d_{1}, i d\right)
$$

Thus

$$
s_{2}\left(\left(e_{0}, z\right)\right)=\left(\left(\mu(z)+e_{0}, y-z+a\right),\left(e_{0}, y\right),\left(e_{0}, z\right)\right)
$$

where $y:=\sigma\left(s \phi\left(e_{0}\right)-e_{0}\right)$ and $a \in A$.

Moreover, one can see that on the underlying object level the following square is a pullback:

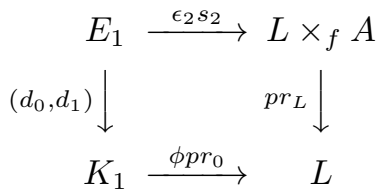

It follows from Definition 2.12 that $(E ., \epsilon$.) is a 2 -torsor over $\mathrm{L}$ relative to $U$, where $\epsilon .: E . \rightarrow K((A, f), 2)$ is the simplicial morphism obtained by the normalized cocycle $\epsilon_{2}$. Also, one can see that the Moore complex $M(E$. of $E$. is the exact sequence

$$
0 \rightarrow A \rightarrow M \stackrel{\mu}{\rightarrow} E_{0} \stackrel{\phi}{\rightarrow} L \rightarrow 0
$$

Notice that a 2 -fold extension in $E^{2}(\phi,(A, f))$ is associated to a 2-torsor over $L$ with augmentation $\phi$. 
If

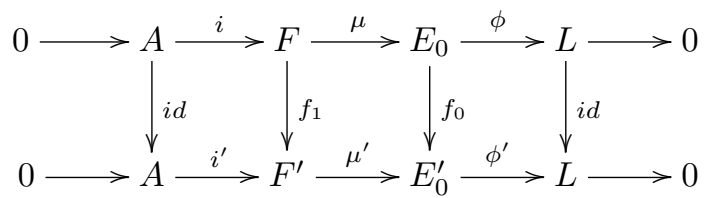

is a morphism of 2-fold extensions, then we obtain a morphism of 2-torsors

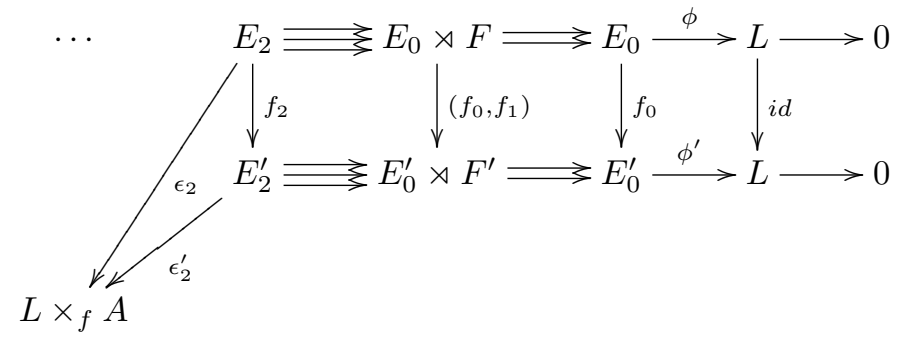

Since we have, for $t=\left(\left(\mu(z)+e_{0}, x\right),\left(e_{0}, y\right),\left(e_{0}, z\right)\right) \in E_{2}$ and $x, y, z \in F$,

$$
\begin{aligned}
\epsilon_{2}^{\prime}\left(f_{2}(t)\right) & =\epsilon_{2}^{\prime}\left(\left(f_{0}\left(\mu(z)+e_{0}\right), f_{1}(x)\right),\left(f_{0}\left(e_{0}\right), f_{1}(y)\right),\left(f_{0}\left(e_{0}\right), f_{1}(z)\right)\right) \\
& =\epsilon_{2}^{\prime}\left(\left(\mu^{\prime}\left(f_{1}(z)\right)+f_{0}\left(e_{0}\right), f_{1}(x)\right),\left(f_{0}\left(e_{0}\right), f_{1}(y)\right),\left(f_{0}\left(e_{0}\right), f_{1}(z)\right)\right) \\
& =\left(\phi^{\prime}\left(f_{0}\left(e_{0}\right)\right), f_{1}(x)+f_{1}(z)-f_{1}(y)\right) \\
& =\left(\phi\left(e_{0}\right), f_{1}(x+z-y)\right) \\
& =\epsilon_{2}\left(\left(\mu(z)+e_{0}, x\right),\left(e_{0}, y\right),\left(e_{0}, z\right)\right) \\
& =\epsilon_{2}(t)
\end{aligned}
$$

thus maps

$$
\omega: \operatorname{Ext}_{p}^{2}(L,(A, f)) \rightarrow \operatorname{Tors}^{2}(L,(A, f))
$$

and

$$
\omega: \operatorname{Ext}_{p}^{2}(\phi,(A, f)) \rightarrow \operatorname{Tors}^{2}(\phi,(A, f))
$$

are induced. Clearly, the map $\psi$ is inverse to $\omega$ and $\psi^{\prime}$ is inverse to $\omega^{\prime}$.

\subsection{Sum of 2-torsors and 2-fold extensions}

First we recall the construction of a sum of torsors given in [15]. Let $\mathcal{E}=(E, \epsilon)$ and $\mathcal{E}^{\prime}=\left(E^{\prime}, \epsilon^{\prime}\right)$ be two 2-torsors over $L$ under $(A, f)$. We consider the product simplicial object $\mathcal{E} \times \mathcal{E}^{\prime}$ formed of products dimension by dimension. Then $\mathcal{E} \times \mathcal{E}^{\prime}$ is a 2 -torsor over $L \times L$ under $(A, f) \times(A, f)$. Let $\Delta$ be the diagonal map $L \rightarrow L \times L$. By Corollary 3.8.3 in [15] a 2-torsor $\Delta^{*}\left(\mathcal{E} \times \mathcal{E}^{\prime}\right)$ over $L$ under $(A, f) \times(A, f)$ is constructed. In particular, in 0 dimension, $\Delta^{*}\left(\mathcal{E} \times \mathcal{E}^{\prime}\right)_{0}$ is obtained by a pullback along $\Delta$ :

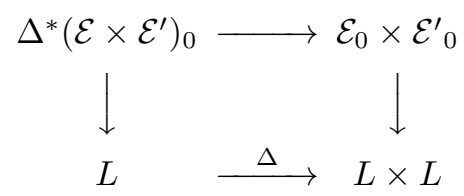


If $\bar{K}$ is the simplicial kernel of $\Delta^{*}\left(\mathcal{E} \times \mathcal{E}^{\prime}\right)_{0} \rightarrow L$ and $K$ is the simplicial kernel of $\mathcal{E}_{0} \times \mathcal{E}^{\prime}{ }_{0} \rightarrow L \times L$, then $\Delta^{*}\left(\mathcal{E} \times \mathcal{E}^{\prime}\right)_{1}$ is the pullback

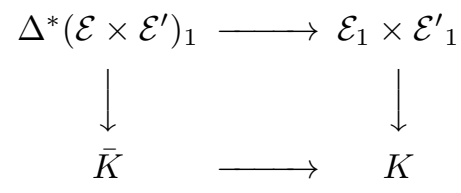

The simplicial object $\Delta^{*}\left(\mathcal{E} \times \mathcal{E}^{\prime}\right)$ is defined (see Proposition 3.8.1 in [15]) to be

$$
\operatorname{cosk}^{1}\left(\Delta^{*}\left(\mathcal{E} \times \mathcal{E}^{\prime}\right)_{1} \longrightarrow \Delta^{*}\left(\mathcal{E} \times \mathcal{E}^{\prime}\right)_{0} \longrightarrow L\right)
$$

The addition homomorphism

$$
\left(L \times_{f} A\right) \times_{L}\left(L \times_{f} A\right) \rightarrow L \times_{f} A
$$

is given by

$$
\nabla:((l, a),(l, b)) \mapsto(l, a+b)
$$

By Proposition 4.3 in [15] (see also Definition 2.4 in $[\mathbf{7}]$ ), the addition homomorphism induces the existence of a 2 -torsor $\nabla_{*}\left(\Delta^{*}\left(\mathcal{E} \times \mathcal{E}^{\prime}\right)\right)$ over $L$ under $(A, f)$ called the sum of $\mathcal{E}$ and $\mathcal{E}^{\prime}$ such that one has a commutative diagram

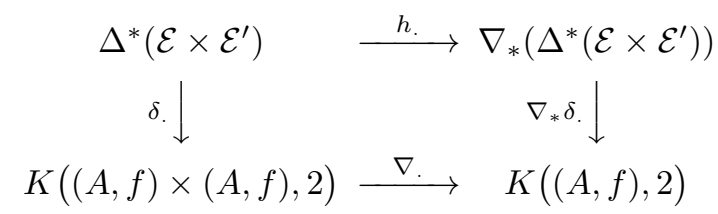

Moreover, the above square has the universal property that if $\mathcal{G}$ is any 2-torsor above $L$ under $(A, f)$ such that

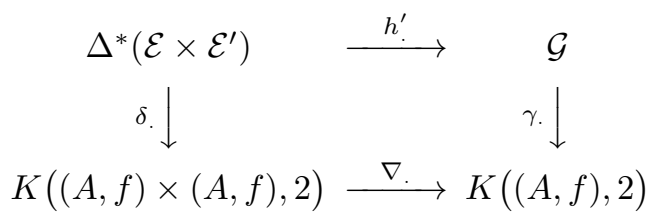

then there exists a unique map

$$
\lambda_{.}: \nabla_{*}\left(\Delta^{*}\left(\mathcal{E} \times \mathcal{E}^{\prime}\right)\right) \rightarrow \mathcal{G}
$$

such that $\gamma . \lambda .=\nabla_{*} \delta$. and $\lambda . h .=h^{\prime}$. The sum of 2-torsors induces a group structure on the set $\operatorname{Tors}_{p}^{2}(L,(A, f))$.

Besides, the Baer sum of two 2-fold extensions and a group structure on $\operatorname{Ext}_{p}^{2}(L,(A, f))$ are defined following the general constructions of Yoneda's theory. Furthermore, the interested reader could also consult [14] (see Definition 5) and [3] (see page 424). Extensions of restricted Lie algebras are discussed in [16]. The Baer sum of 2-fold extensions of restricted Lie algebras is induced by their sum as 2-fold extensions of Lie algebras. 
Let

$$
(E): \quad 0 \rightarrow A \stackrel{i}{\rightarrow} F \stackrel{\mu}{\rightarrow} E \stackrel{\phi}{\rightarrow} L \rightarrow 0
$$

and

$$
\left(E^{\prime}\right): \quad 0 \rightarrow A \stackrel{i^{\prime}}{\rightarrow} F^{\prime} \stackrel{\mu^{\prime}}{\rightarrow} E^{\prime} \stackrel{\phi^{\prime}}{\rightarrow} L \rightarrow 0
$$

be two extensions in $E^{2}(L,(A, f))$. Then the Baer sum of $(E)$ and $\left(E^{\prime}\right)$ is defined as the extension

$$
\left(E+E^{\prime}\right): \quad 0 \rightarrow(A \times A) / \mathcal{K} \rightarrow\left(F \times F^{\prime}\right) / \mathcal{K} \rightarrow E \times{ }_{L} E^{\prime} \rightarrow L \rightarrow 0
$$

where $\mathcal{K}:=\operatorname{ker} \nabla$ and $\nabla: A \times A \rightarrow A$ is given by $\nabla\left(a, a^{\prime}\right):=a+a^{\prime}$ for all $a, a^{\prime} \in A$. The restricted ideal $\mathcal{K}$ consists of the elements $(a,-a)$. We identify $(A \times A) / \mathcal{K}$ with $A$ and we get

$$
\left(E+E^{\prime}\right): \quad 0 \rightarrow A \rightarrow\left(F \times F^{\prime}\right) /\{(a,-a): a \in A\} \rightarrow E \times{ }_{L} E^{\prime} \rightarrow L \rightarrow 0
$$

Theorem 5.2. Let $L \in$ RLie be a restricted Lie algebra and $(A, f)$ a $w(L)$-module. Then there is an isomorphism of groups

$$
\operatorname{Tors}^{2}(L,(A, f)) \simeq \operatorname{Ext}_{p}^{2}(L,(A, f))
$$

Proof. Let $E, E^{\prime} \in E^{2}(L,(A, f))$ be two extensions

$$
0 \rightarrow A \rightarrow F \stackrel{\mu}{\rightarrow} E_{0} \stackrel{\phi}{\rightarrow} L \rightarrow 0
$$

and

$$
0 \rightarrow A \rightarrow F^{\prime} \stackrel{\mu^{\prime}}{\rightarrow} E_{0}^{\prime} \stackrel{\phi^{\prime}}{\longrightarrow} L \rightarrow 0
$$

with associated 2-torsors

$$
\omega(E):=\operatorname{cosk}^{1}\left(\left(E_{0} \rtimes F\right) \Longrightarrow E_{0} \longrightarrow L\right)
$$

and

$$
\omega\left(E^{\prime}\right):=\operatorname{cosk}^{1}\left(\left(E_{0}^{\prime} \rtimes F^{\prime}\right) \longrightarrow E_{0}^{\prime} \longrightarrow L\right)
$$

We recall that the sum $\left(E+E^{\prime}\right)$ of the extensions $(E)$ and $\left(E^{\prime}\right)$ is given by

$$
\left(E+E^{\prime}\right): \quad 0 \rightarrow A \rightarrow\left(F \times F^{\prime}\right) /\{(a,-a): a \in A\} \rightarrow E_{0} \times_{L} E_{0}^{\prime} \rightarrow L \rightarrow 0
$$

From the definition of $\Delta^{*}\left(\omega(E) \times \omega\left(E^{\prime}\right)\right)$ we have that

$$
\Delta^{*}\left(\omega(E) \times \omega\left(E^{\prime}\right)\right)_{0} \simeq E_{0} \times_{L} E_{0}^{\prime}
$$

and $\Delta^{*}\left(\omega(E) \times \omega\left(E^{\prime}\right)\right)_{1}$ is the pullback

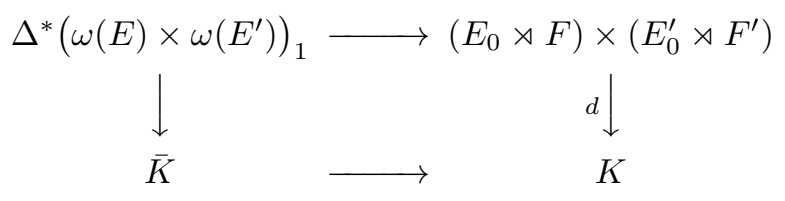

where $\bar{K}$ is the simplicial kernel

$$
\bar{K} \Longrightarrow E_{0} \times_{L} E_{0}^{\prime} \longrightarrow L
$$


and $K$ is the simplicial kernel

$$
K \Longrightarrow E_{0} \times E_{0}^{\prime} \longrightarrow L \times L
$$

Thus

$$
\begin{aligned}
\bar{K}:=\left\{\left(\left(e_{0}, e_{0}^{\prime}\right),\left(e_{0}+\mu(x), e_{0}^{\prime}+\mu^{\prime}\left(x^{\prime}\right)\right):\right.\right. & \phi\left(e_{0}\right)=\phi^{\prime}\left(e_{0}^{\prime}\right), \\
& \left.e_{0} \in E_{0}, e_{0}^{\prime} \in E_{0}^{\prime}, x \in F, x^{\prime} \in F^{\prime}\right\}
\end{aligned}
$$

and

$$
K:=\left\{\left(\left(e_{0}, e_{0}^{\prime}\right),\left(e_{0}+\mu(x), e_{0}^{\prime}+\mu^{\prime}\left(x^{\prime}\right)\right): e_{0} \in E_{0}, e_{0}^{\prime} \in E_{0}^{\prime}, x \in F, x^{\prime} \in F^{\prime}\right\}\right.
$$

The canonical morphism $d$ is given by

$$
d\left(\left(e_{0}, x\right),\left(e_{0}^{\prime}, x^{\prime}\right)\right)=\left(\left(e_{0}+\mu(x), e_{0}^{\prime}+\mu^{\prime}\left(x^{\prime}\right)\right),\left(e_{0}, e_{0}^{\prime}\right)\right)
$$

Therefore

$$
\begin{aligned}
\Delta^{*}(\omega(E) & \left.\times \omega\left(E^{\prime}\right)\right)_{1}= \\
= & \left\{\left(\left(e_{0}, e_{0}^{\prime}\right),\left(e_{0}+\mu(x), e_{0}^{\prime}+\mu^{\prime}\left(x^{\prime}\right)\right),\left(e_{0}+\mu(x), a-x\right),\left(e_{0}^{\prime}+\mu^{\prime}(x), a^{\prime}-x^{\prime}\right)\right):\right. \\
& \left.\phi\left(e_{0}\right)=\phi^{\prime}\left(e_{0}^{\prime}\right), x \in F, x^{\prime} \in F^{\prime}, a, a^{\prime} \in A, e_{0} \in E_{0}, e_{0}^{\prime} \in E_{0}\right\}
\end{aligned}
$$

There is a natural morphism of truncated simplicial objects

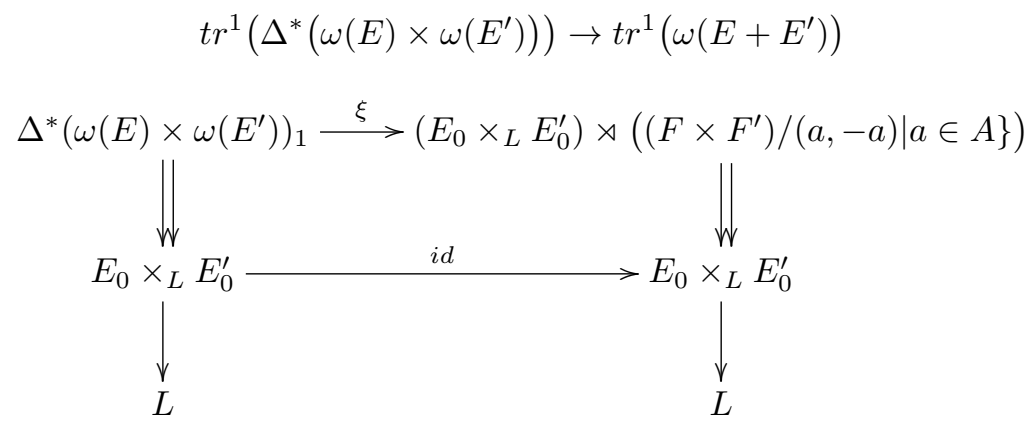

where $\xi$ is given by

$$
\begin{array}{r}
\xi\left(\left(e_{0}, e_{0}^{\prime}\right),\left(e_{0}+\mu(x), e_{0}^{\prime}+\mu^{\prime}\left(x^{\prime}\right)\right),\left(e_{0}+\mu(x), a-x\right),\left(e_{0}^{\prime}+\mu^{\prime}(x), a^{\prime}-x^{\prime}\right)\right)= \\
=\left(\left(e_{0}+\mu(x), e_{0}^{\prime}+\mu^{\prime}\left(x^{\prime}\right)\right), \overline{\left(a-x, a^{\prime}-x^{\prime}\right)}\right)
\end{array}
$$

Next we prove that $\xi$ is a Lie algebra homomorphism. Let $e_{0}, h_{0} \in E_{0}$ and $x, y \in F$. If we set $t:=e_{0} \cdot y-h_{0} \cdot x+[x, y]$, then

$$
\begin{aligned}
{\left[e_{0}+\mu(x), h_{0}+\mu(y)\right] } & =\left[e_{0}, h_{0}\right]+\left[e_{0}, \mu(y)\right]+\left[\mu(x), h_{0}\right]+[\mu(x), \mu(y)] \\
& =\left[e_{0}, h_{0}\right]+\mu\left(e_{0} \cdot y-h_{0} \cdot x+[x, y]\right) \\
& =\left[e_{0}, h_{0}\right]+\mu(t)
\end{aligned}
$$

and

$$
\begin{aligned}
& {\left[\left(e_{0}+\mu(x), a_{0}-x\right),\left(h_{0}+\mu(y), a_{1}-y\right)\right]=} \\
& \quad=\left(\left[e_{0}, h_{0}\right]+\mu(t),\left(e_{0}+\mu(x)\right) \cdot\left(a_{1}-y\right)-\left(h_{0}+\mu(y)\right) \cdot\left(a_{0}-x\right)+[x, y]\right) \\
& \quad=\left(\left[e_{0}, h_{0}\right]+\mu(t), e_{0} \cdot a_{1}-h_{0} \cdot a_{0}-t\right)
\end{aligned}
$$


Let $x_{1}, y_{1} \in \Delta^{*}\left(\omega(E) \times \omega\left(E^{\prime}\right)\right)_{1}$, where

$$
\begin{aligned}
& x_{1}:=\left(\left(e_{0}, e_{0}^{\prime}\right),\left(e_{0}+\mu(x), e_{0}^{\prime}+\mu^{\prime}\left(x^{\prime}\right)\right),\left(e_{0}+\mu(x), a_{0}-x\right),\left(e_{0}^{\prime}+\mu^{\prime}(x), a_{0}^{\prime}-x^{\prime}\right)\right) \\
& y_{1}:=\left(\left(h_{0}, h_{0}^{\prime}\right),\left(h_{0}+\mu(y), h_{0}^{\prime}+\mu^{\prime}\left(y^{\prime}\right)\right),\left(h_{0}+\mu(y), a_{1}-y\right),\left(h_{0}^{\prime}+\mu^{\prime}(y), a_{1}^{\prime}-y^{\prime}\right)\right)
\end{aligned}
$$

If we set $t^{\prime}:=e_{0}^{\prime} \cdot y^{\prime}-h_{0}^{\prime} \cdot x^{\prime}+\left[x^{\prime}, y^{\prime}\right]$, then we have

$$
\begin{aligned}
\xi\left(\left[\left(x_{1}, y_{1}\right)\right]\right)= & \\
= & \xi\left(\left(\left[e_{0}, h_{0}\right],\left[e_{0}^{\prime}, h_{0}^{\prime}\right]\right),\left(\left[e_{0}, h_{0}\right]+\mu(t),\left[e_{0}^{\prime}, h_{0}^{\prime}\right]+\mu^{\prime}\left(t^{\prime}\right)\right),\left(\left[e_{0}, h_{0}\right]+\mu(t),\right.\right. \\
& \left.\left.e_{0} \cdot a_{1}-h_{0} \cdot a_{0}-t\right),\left(\left[e_{0}^{\prime}, h_{0}^{\prime}\right]+\mu\left(t^{\prime}\right), e_{0}^{\prime} \cdot a_{1}^{\prime}-h_{0}^{\prime} \cdot a_{0}^{\prime}-t^{\prime}\right)\right) \\
= & \left.\left(\left[e_{0}, h_{0}\right]+\mu(t),\left[e_{0}^{\prime}, h_{0}^{\prime}\right]+\mu^{\prime}\left(t^{\prime}\right)\right), \overline{\left(e_{0} \cdot a_{1}-h_{0} \cdot a_{0}-t, e_{0}^{\prime} \cdot a_{1}^{\prime}-h_{0}^{\prime} \cdot a_{0}^{\prime}-t^{\prime}\right)}\right) \\
= & {\left[\left(\left(e_{0}+\mu(x), e_{0}^{\prime}+\mu^{\prime}\left(x^{\prime}\right)\right), \overline{\left(a_{0}-x, a_{0}^{\prime}-x^{\prime}\right)}\right),\right.} \\
& \left.\left.\left(\left(h_{0}+\mu(y), h_{0}^{\prime}+\mu^{\prime}\left(y^{\prime}\right)\right), \overline{\left(a_{1}-y, a_{1}^{\prime}-y^{\prime}\right.}\right)\right)\right]
\end{aligned}
$$

Therefore $\xi$ is a Lie algebra homomorphism. Besides, one can easily see that

$$
\xi\left(\left(\left(e_{0}, e_{0}^{\prime}\right),\left(e_{0}, e_{0}^{\prime}\right),\left(e_{0}, 0\right),\left(e_{0}^{\prime}, 0\right)\right)^{[p]}\right)=\left(\xi\left(\left(e_{0}, e_{0}^{\prime}\right),\left(e_{0}, e_{0}^{\prime}\right),\left(e_{0}, 0\right),\left(e_{0}^{\prime}, 0\right)\right)\right)^{[p]}
$$

Since $[a, x]=0$ and $\mu(x) \cdot(a-x)=0$, we have

$$
\begin{gathered}
(\mu(x), a-x)^{[p]}=\left(\mu\left(x^{[p]}\right), a^{[p]}-x^{[p]}\right) \\
\xi\left(\left((0,0),\left(\mu(x), \mu^{\prime}\left(x^{\prime}\right)\right),(\mu(x), a-x),\left(\mu^{\prime}(x), a^{\prime}-x^{\prime}\right)\right)^{[p]}\right)= \\
\left(\xi\left((0,0),\left(\mu(x), \mu^{\prime}\left(x^{\prime}\right)\right),(\mu(x), a-x),\left(\mu^{\prime}(x), a^{\prime}-x^{\prime}\right)\right)\right)^{[p]}
\end{gathered}
$$

It follows that $\xi$ is a restricted Lie homomorphism. By applying the coskeleton functor $\operatorname{cosk}^{1}(-)$ to the truncated simplicial complexes, a morphism is induced:

$$
\xi .:\left(\Delta^{*}\left(\omega(E) \times \omega\left(E^{\prime}\right)\right) \rightarrow \omega\left(E+E^{\prime}\right)\right.
$$

Next we prove that we have the following commutative diagram

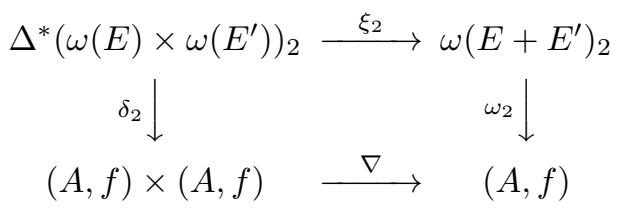

Let $\left(k_{0}, k_{1}, k_{2}\right) \in \Delta^{*}\left(\omega(E) \times \omega\left(E^{\prime}\right)\right)_{2}$ where

$$
\begin{aligned}
k_{0}:= & \left(\left(e_{0}-\mu(y), e_{0}^{\prime}-\mu^{\prime}\left(y^{\prime}\right)\right),\left(e_{0}, e_{0}^{\prime}\right),\left(e_{0}, a_{0}-y\right),\left(e_{0}^{\prime}, a_{0}^{\prime}-y^{\prime}\right)\right) \\
k_{1}:= & \left(\left(e_{0}-\mu(y), e_{0}^{\prime}-\mu^{\prime}\left(y^{\prime}\right)\right),\left(e_{0}+\mu(x), e_{0}^{\prime}+\mu^{\prime}\left(x^{\prime}\right)\right),\left(e_{0}+\mu(x), a_{1}-(x+y)\right),\right. \\
\quad & \left.\left(e_{0}^{\prime}+\mu^{\prime}(x), a_{1}^{\prime}-\left(x^{\prime}+y^{\prime}\right)\right)\right) \\
k_{2}:= & \left(\left(e_{0}, e_{0}^{\prime}\right),\left(e_{0}+\mu(x), e_{0}^{\prime}+\mu^{\prime}\left(x^{\prime}\right)\right),\left(e_{0}+\mu(x), a_{2}-x\right),\left(e_{0}^{\prime}+\mu^{\prime}(x), a_{2}^{\prime}-x^{\prime}\right)\right)
\end{aligned}
$$


Then by definition of the normalized cocycle $\delta_{2}$ we obtain

$$
\begin{aligned}
\nabla \delta_{2}\left(k_{0}, k_{1}, k_{2}\right)=\nabla\left(\left(\phi\left(e_{0}\right),\left(a_{0}-y\right)\right.\right. & \left.+\left(a_{2}-x\right)-\left(a_{1}-(x+y)\right)\right), \\
& \left.\left(\phi^{\prime}\left(e_{0}^{\prime}\right),\left(a_{0}^{\prime}-y^{\prime}\right)+\left(a_{2}^{\prime}-x^{\prime}\right)-\left(a_{1}^{\prime}-\left(x^{\prime}+y^{\prime}\right)\right)\right)\right)
\end{aligned}
$$

Hence,

$$
\nabla \delta_{2}\left(k_{0}, k_{1}, k_{2}\right)=\left(\phi\left(e_{0}\right),\left(a_{0}+a_{2}-a_{1}\right)+\left(a_{0}^{\prime}+a_{2}^{\prime}-a_{1}^{\prime}\right)\right)
$$

Besides,

$$
\begin{aligned}
\omega_{2} \xi_{2}\left(k_{0}, k_{1}, k_{3}\right) & \\
= & \omega_{2}\left(\left(\left(e_{0}, e_{0}^{\prime}\right), \overline{\left(a_{0}-y, a_{0}^{\prime}-y^{\prime}\right.}\right)\right),\left(\left(e_{0}+\mu(x), e_{0}^{\prime}+\mu^{\prime}\left(x^{\prime}\right),\right.\right. \\
& \left.\left.\left.\left.\overline{\left(a_{1}-(x+y), a_{1}^{\prime}-\left(x^{\prime}+y^{\prime}\right)\right.}\right)\right),\left(\left(e_{0}+\mu(x), e_{0}^{\prime}+\mu^{\prime}\left(x^{\prime}\right)\right), \overline{\left(a_{2}-x, a_{2}^{\prime}-x^{\prime}\right.}\right)\right)\right)
\end{aligned}
$$

Thus

$$
\omega_{2} \xi_{2}\left(k_{0}, k_{1}, k_{3}\right)=\left(\phi\left(e_{0}\right),\left(a_{0}+a_{2}-a_{1}\right)+\left(a_{0}^{\prime}+a_{2}^{\prime}-a_{1}^{\prime}\right)\right)
$$

It follows that

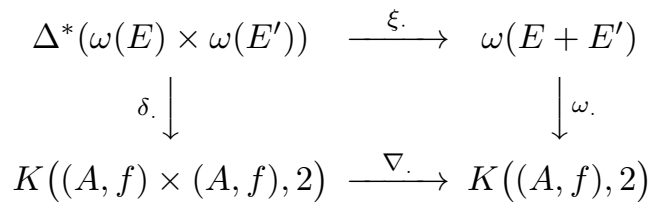

From the universal property of $\nabla_{*} \Delta^{*}\left(\omega(E) \times \omega\left(E^{\prime}\right)\right)$ (see Subsection 5.2), there exists a unique map

$$
\lambda .: \nabla_{*} \Delta^{*}\left(\omega(E) \times \omega\left(E^{\prime}\right)\right) \rightarrow \omega\left(E+E^{\prime}\right)
$$

Thus $\nabla_{*} \Delta^{*}\left(\omega(E) \times \omega\left(E^{\prime}\right)\right)$ and $\omega\left(E+E^{\prime}\right)$ are in the same component.

Remark 5.3. From Proposition 2.6 in $[\mathbf{7}]$, Tors ${ }^{1}(\phi,(A, f))$ is an abelian group and by Lemma 5.1 this is transferred to a group structure on the set $\operatorname{Ext}_{p}^{2}(\phi,(A, f))$.

Theorem 5.4. Let $L \in \mathrm{RLie}$ be a restricted Lie algebra and $(A, f)$ a $w(L)$-module. Then

$$
H_{\mathbf{G}}^{2}(L,(A, f)) \simeq \operatorname{Ext}_{p}^{2}(L,(A, f))
$$

Proof. This theorem follows from Theorem 5.2 and Theorem 2.13.

Remark 5.5. We note that in the Cartan-Eilenberg context, crossed modules in various algebraic categories are associated to the third cohomology group. In the QuillenBarr-Beck context, crossed modules are associated to the second cohomology group since there is a shift by 1 in the notation. Besides, G. Hochschild in [17] gives an interpretation of the third Hochschild cohomology in terms of space of restricted kernel classes.

\section{3. $\quad$ Eight-term exact sequence}

The five-term exact sequence of Theorem 3.2 for Quillen-Barr-Beck cohomology for restricted Lie algebras can be extended to an eight-term exact sequence by the following theorem. 
Theorem 5.6. Let $0 \rightarrow N \rightarrow E \stackrel{\phi}{\rightarrow} L \rightarrow 0$ be an exact sequence of restricted Lie algebras and let $(A, f)$ be a $w(L)$-module. Then there are naturally equivalent exact sequences

$$
\begin{gathered}
0 \rightarrow \operatorname{Der}_{p}(L,(A, f)) \rightarrow \operatorname{Der}_{p}(E,(A, f)) \rightarrow \operatorname{Hom}_{w(L)}\left(\left(N_{a b},(-)^{[p]}\right),(A, f)\right) \rightarrow \\
\operatorname{Ext}_{p}^{1}(L,(A, f)) \rightarrow \rightarrow \operatorname{Ext}_{p}^{1}(E,(A, f)) \rightarrow \operatorname{Ext}_{p}^{2}(\phi,(A, f)) \rightarrow \\
\operatorname{Ext}_{p}^{2}(L,(A, f)) \rightarrow \operatorname{Ext}_{p}^{2}(E,(A, f)) \\
0 \rightarrow H_{\mathbf{G}}^{0}(L,(A, f)) \rightarrow H_{\mathbf{G}}^{0}(E,(A, f)) \rightarrow \operatorname{Hom}_{w(L)}\left(\left(N_{a b},(-)^{[p]}\right),(A, f)\right) \rightarrow \\
H_{G}^{1}(L,(A, f)) \rightarrow \rightarrow H_{\mathbf{G}}^{1}(E,(A, f)) \rightarrow \operatorname{Ext}_{p}^{2}(\phi,(A, f)) \rightarrow \\
H_{\mathbf{G}}^{2}(L,(A, f)) \rightarrow H_{\mathbf{G}}^{2}(E,(A, f))
\end{gathered}
$$

Proof. This theorem follows from Theorem 5.4 and the eight-term exact sequence (5).

\subsection{Application to extensions of algebraic groups}

Let $k$ be an algebraic closed field of prime characteristic and $\mathrm{G}$ an algebraic group over $k$. Since $\operatorname{char} k=p$, we have that the associated Lie algebra $\operatorname{Lie}(\mathrm{G})$ is actually a restricted Lie algebra. In fact in this way a functor Lie $: \mathcal{G} r \rightarrow$ RLie is defined from the category of algebraic groups to the category of restricted Lie algebras (see [4]).

Let K, G be algebraic groups; then M. Rosenlicht in [25] and J.-P. Serre in [26] define as an extension of $\mathrm{G}$ by $\mathrm{K}$ a short exact sequence of groups

$$
0 \rightarrow \mathrm{K} \stackrel{\kappa}{\rightarrow} \mathrm{H} \stackrel{\nu}{\rightarrow} \mathrm{G} \rightarrow 0
$$

such that $\kappa, \nu$ are separable rational homomorphisms.

Proposition 5.7. Let $0 \rightarrow \mathrm{K} \rightarrow \mathrm{H} \stackrel{\nu}{\rightarrow} \mathrm{G} \rightarrow 0$ be an exact sequence of algebraic groups and $(A, f)$ a $w(\operatorname{Lie}(\mathrm{G}))$-module. Then the following sequence is exact:

$$
\begin{aligned}
& 0 \rightarrow \operatorname{Der}_{p}(\operatorname{Lie}(\mathrm{G}),(A, f)) \rightarrow \operatorname{Der}_{p}(\operatorname{Lie}(\mathbf{H}),(A, f)) \rightarrow \\
& \operatorname{Hom}_{w(\operatorname{Lie}(\mathbf{G}))}\left(\left(\operatorname{Lie}(\mathrm{K})_{a b},(-)^{[p]}\right),(A, f)\right) \rightarrow H_{\mathbf{G}}^{1}(\operatorname{Lie}(\mathrm{G}),(A, f)) \rightarrow \\
& H_{\mathbf{G}}^{1}(\operatorname{Lie}(\mathrm{H}),(A, f)) \rightarrow \operatorname{Ext}_{p}^{2}(\operatorname{Lie}(\nu),(A, f)) \rightarrow \\
& H_{\mathbf{G}}^{2}(\operatorname{Lie}(\mathrm{G}),(A, f)) \rightarrow H_{\mathbf{G}}^{2}(\operatorname{Lie}(\mathbf{H}),(A, f))
\end{aligned}
$$

Proof. Since the induced sequence of restricted Lie algebras

$$
0 \rightarrow \operatorname{Lie}(\mathrm{K}) \rightarrow \operatorname{Lie}(\mathrm{H}) \stackrel{\operatorname{Lie}(\nu)}{\longrightarrow} \operatorname{Lie}(\mathrm{G}) \rightarrow 0
$$

is exact (see [25]), the result follows from Theorem 5.6.

\section{Acknowledgments}

I would like to thank the anonymous referee for very valuable comments which provided insights that helped to considerably improve the paper. 


\section{References}

[1] M. Barr, Cartan-Eilenberg cohomology and triples, Journal of Pure and Applied Algebra 112 (1996), 219-238.

[2] M. Barr and J. Beck, Homology and standard constructions, Seminar on Triples and Categorical Homology Theory, vol. 80, Springer-Verlag, Berlin, 1969, 245-335.

[3] M. Barr and G. Rinehart, Cohomology as the derived functor of derivations, Trans. Amer. Math. Soc. 122 (1966), 416-426.

[4] A. Borel, Linear algebraic groups, Graduate Texts in Mathematics, SpringerVerlag, New York, 1991.

[5] R. Brown and C.B. Spencer, G-groupoids, crossed modules and the fundamental groupoid of a topological group, Proc. Kon. Ned. Acad. 79 (1976), 296-302.

[6] H. Cartan and S. Eilenberg, Homological Algebra, Princeton University Press, Princeton, 1956.

[7] A.M. Cegarra and E.R Aznar, An exact sequence in the first variable for torsor cohomology: The 2-dimensional theory of obstructions, Journal of Pure and Applied Algebra 39 (1986), 197-250.

[8] I. Dokas, A (co-) homology for restricted Lie algebras, Thesis, University of Warwick, 2000.

[9] I. Dokas, Quillen-Barr-Beck (co-) homology for restricted Lie algebras, Journal of Pure and Applied Algebra 186 (2004), 33-42.

[10] J. Duskin, Simplicial methods and the interpretation of Triple Cohomology, Mem. Amer. Math. Soc. issue 2, 163, 1975.

[11] J. Duskin, Higher-dimensional torsors and the cohomology of topoi: the abelian theory, Applications of sheaves (Proc. Res. Sympos. Appl. Sheaf Theory to Logic, Algebra and Anal., Univ. Durham, Durham, 1977), Lecture Notes in Math., 753, Springer, Berlin, 1979, 255-279.

[12] B. Eckmann and U. Stammbach, On exact sequences in the homology of groups and algebras, Illinois J. Math. 14 (1970), 205-215.

[13] G.J. Ellis, A non-abelian tensor product of Lie algebras, Glasgow Math. J. 33 (1991), 101-120.

[14] M. Gerstenhaber, On the deformation of rings and algebras II. Ann. of Math. 84 (1966), 1-19.

[15] P.G. Glenn, Realization of cohomology classes in arbitrary exact Categories, Journal of Pure and Applied Algebra 25 (1982), 33-105.

[16] G. Hochschild, Cohomology of restricted Lie algebras, Amer. J. Math. 76 (1954), 555-580.

[17] G. Hochschild, Lie algebra kernels and cohomology, Amer. J. Math. 76 (1954), 698-716.

[18] N. Jacobson, Lie algebras, Dover Publications, Inc., New York, 1979.

[19] G. Janelidze and M.C Pedicchio, Internal Categories and Groupoids in Congruence Modular Varieties, Journal of Algebra 193 (1997), 552-570. 
[20] C. Kassel and J.-L. Loday, Extensions centrales d' algebres de Lie, Ann. Inst. Fourier 32 (1982), 119-142.

[21] B. Pareigis, Cohomologie des p-algèbres de Lie, C. R. Acad. Sci. Paris Ser. $A-B 263$ (1966), 709-712.

[22] T. Porter, Crossed modules in Cat and a Brown-Spencer theorem for 2categories, Cahiers Topologie Géom. Différentielle Catég. 26 (1985), 381-388.

[23] D. Quillen, On the (co-) homology of commutative rings, Proceedings of Symposium on Pure Mathematics, Vol XVII, AMS, Providence, RI, 1970.

[24] D. Quillen, Homotopical algebra, Lecture Notes in Mathematics, 43, SpringerVerlag, Berlin-New York, 1967.

[25] M. Rosenlicht, Extensions of vector groups by abelian varieties, Amer. J. Math. 80 (1958), 685-714.

[26] J.-P. Serre, Quelques propriétés des variétés abéliennes en caractéristique $p$, Amer. J. Math. 80 (1958), 715-739.

[27] N. Shimada, H. Uehara, F. Brenneman, and A. Iwai, Triple cohomology of algebras and two term extensions, Publ. Res. Inst. Math. Sci. 5 (1969), 267285.

[28] H. Strade and R. Farnsteiner, Modular Lie algebras and their representations, Marcel Dekker, 1988.

[29] M. Vale, Torsors and special extensions, Cahiers Topologie Géom. Différentielle Catég. 26 (1985), 63-90.

[30] W.C. Waterhouse, Introduction to Affine Group Schemes, Springer Verlag, New York, 1979.

[31] J.H.C. Whitehead, Combinatorial homotopy, II, Bull. Amer. Math. Soc. 5 (1949), 453-496.

Ioannis Dokas dokas@ucy.ac.cy

Department of Mathematics and Statistics, University Of Cyprus, P.O. Box 20537, CY-1678 Nicosia, Cyprus 